THEP 02/06

University of Freiburg

7th June 2002

\title{
The Invariant Charges of the Nambu-Goto String Theory: Quantization of Non-Additive Composition Laws
}

\author{
K. Pohlmeyer and M. Trunk \\ Fakultät für Physik der Universität Freiburg, Hermann-Herder-Str. 3, \\ D-79104 Freiburg, Germany
}

\begin{abstract}
We examine and implement the concept of non-additive composition laws in the quantum theory of closed bosonic strings moving in $(3+1)$-dimensional Minkowski space. Such laws supply exact selection rules for the merging and splitting of closed strings.
\end{abstract}




\section{Introduction}

A relevant part of the quantum algebra formed by the infinitesimal generators of observable symmetry transformations for the massive relativistic closed bosonic string moving in $(3+1)$-dimensional space-time has been constructed and analyzed Ref. [1]. The correspondence principle being used as a guide, a thorough understanding of the observable features of the classical theory proved to be basic for the presentation of the quantum theory. However, not all observable structures of the classical theory identified so far have been implemented in the quantum theory. One particular such structure of the classical theory, the laws for the merging of two closed strings in a single such string and, vice versa, for the splitting of a single closed string into two closed strings, or rather the quantization of these laws, is the topic of the present communication.

The laws alluded to expand the Huygens-Newtonian laws of impact for the collision of two freely moving bodies. In either case, after some arithmetics, information about the incoming state of the system produces partial information about the resulting state after the merging or the splitting of the string(s) and after the collision, respectively. In contrast to the Huygens-Newtonian laws of impact, the laws for the string are non-additive in general. Examples of such classical non-additive composition or decomposition laws for the closed strings moving in $(2+1)$-dimensional space-time can be found in Ref. [2]. There a basic strategy - also valid for higher dimensional space-time - has been described on how to trace and determine such laws. Along those lines, J. Großer has specified the first genuinely non-additive composition laws for the merging of two closed strings moving in $(3+1)$-dimensional space-time Ref. [3].

We shall concentrate on these particular classical composition laws and show that all (pre-) conditions for their implementation in the corresponding quantum theory are satisfied, most important, their adherence to the isomorphic generalized commutation relations for the string branches involved.

\section{Classical Theory Part One}

We consider a single closed bosonic massive Nambu-Goto string which forms an infinitely differentiable space-like closed curve without double points in $(3+1)$-dimensional Minkowski space-time and which is furnished with a smooth bundle of planes tangent to its trajectory surface, one plane for every point on the curve. Let its rest mass be denoted by the symbol $\mathfrak{m}: \mathfrak{m}>0$.

As long as the string doesn't develop double points and doesn't make contact with other closed strings which might be around, it sweeps over a two-dimensional smooth tubeshaped branch of its world-surface. At every point of this branch the corresponding tangent space contains both time-like and space-like vectors. In order to parametrize the smooth tube-shaped branch of the string world-surface, we choose a foliation of the Minkowski space by smooth space-like hypersurfaces (labeled by a forward time-like parameter $\tau$ ) intersecting the tube-shaped branch in space-like smooth closed curves $\mathcal{C}_{\tau}$ without double points. The closed curves $\mathcal{C}_{\tau}$ are the integral curves of a smooth space-like vector field $\partial_{\sigma}$ on the tube-shaped branch. The additional choice of a smooth forward time-like vector field $\partial_{\tau}$ on the branch and an explicit $\sigma$-parametrization of a single curve $\mathcal{C}_{\tau_{0}}$ complete the construction. 
Each space-like leaf of the foliation with label $\tau$ carries a Riemannian metric induced from the Minkowski space. It also contains the closed curve $\mathcal{C}_{\tau}$. Inscribe into $\mathcal{C}_{\tau}$ within the leaf with label $\tau$ the minimal surface of least area ( with respect to the Riemannian metric ). An inward and outward directed normal vector field on $\mathcal{C}_{\tau}$ tangent to this minimal surface can be defined. This implies that an inside and an outside, hence an orientation of the branch, can be defined: the parametrization of the branch is said to have positive orientation if the smooth coordinate vector fields $\partial_{\tau}$ and $\partial_{\sigma}$ (in this order!) at any common point of the branch follow each other clockwise on the outer surface.

The infinitesimal generators of observable symmetry transformations of this system form a Poisson algebra. Information about the numerical values of all elements of this algebra - provided certain compatibility requirements are met - permits to reconstruct the tube-shaped branch apart from its position in the direction of the energy-momentum vector of the string. The algebra itself has the following structure: the Poisson Lie algebra of the infinitesimal generators of external (rigid) Poincaré transformations acts on the Poisson combinatorial $*$-algebra $\mathfrak{h}$ of the infinitesimal generators of internal (flexing) symmetry transformations. It is sufficient to specify the algebra $\mathfrak{h}$ in the momentum rest frame of the string. The algebra $\mathfrak{h}$ turns out to be the sum of two Poisson commuting $*$-subalgebras $\mathfrak{h}^{+}$and $\mathfrak{h}^{-}$which are isomorphic up to a global sign in the "structure constants". Thus it suffices to present just one of the two subalgebras, say $\mathfrak{h}^{-}$, the algebra built from the "right-movers", i.e. the right-moving combination of the canonical string variables: $u_{\mu}^{-}(\tau, \sigma):=p_{\mu}(\tau, \sigma)-\frac{1}{2 \pi \alpha^{\prime}} \partial_{\sigma} x_{\mu}(\tau, \sigma)$, the symbol $\alpha^{\prime}$ denoting the inverse string tension. The elements of $\mathfrak{h}^{-}$are obtained from the eigenvalues of a parameter-dependent monodromy matrix corresponding to a system of linear partial differential equations (containing infinitely many free parameters). The integrability conditions of this system are equivalent to the equations of motion for the right-movers.

The algebra, so obtained, is graded under the Poisson bracket operation $\{\cdot, \cdot\}:=2 \pi \alpha^{\prime}$ times the canonical Poisson bracket operation:

$$
\mathfrak{h}^{-}=\bigoplus_{l=0}^{\infty} \mathfrak{V}^{l}, \quad\left\{\mathfrak{V}^{l_{1}}, \mathfrak{V}^{l_{2}}\right\} \subset \mathfrak{V}^{l_{1}+l_{2}}, \quad \mathfrak{V}^{l_{1}} \cdot \mathfrak{V}^{l_{2}} \subset \mathfrak{V}^{l_{1}+l_{2}+1}, \quad l_{1}, l_{2} \in \mathbb{N}_{0}
$$

Moreover, the finite dimensional vector spaces $\mathfrak{V}^{l}$ are invariant under the parity transformation. Hence, they are decomposable into parity eigenspaces

$$
\mathfrak{V}^{l}=\mathfrak{V}_{+}^{l} \oplus \mathfrak{V}_{-}^{l} .
$$

The vector space $\mathfrak{V}^{0}$ consists of parity even elements only: $\mathfrak{V}^{0} \equiv \mathfrak{V}_{+}^{0}$. It is threedimensional and forms a subalgebra isomorphic to the Lie algebra so(3) with generators $J_{1,-1}, J_{1,0}$ and $J_{1,+1}$ in an angular momentum basis, or rather a spin basis. Each one of the vector spaces $\mathfrak{V}_{ \pm}^{l}$ is invariant under the Poisson action of $\mathfrak{V}^{0}$. Hence each $\mathfrak{V}_{ \pm}^{l}$ carries a linear representation of $s o(3)$ and can be decomposed into a direct sum of isotypical components corresponding to the spin $j \in \mathbb{N}_{0}$

$$
\mathfrak{V}_{+}^{l}=\bigoplus_{j=0}^{l+1} \mathfrak{V}_{j,+}^{l}, \quad \mathfrak{V}_{-}^{l}=\bigoplus_{j=0}^{l+1} \mathfrak{V}_{j,-}^{l}
$$

Some of the isotypical components may be trivial. 
In the following we shall only be concerned with the vector spaces $\mathfrak{V}^{l}$ of degree $0 \leq l \leq 2$. Their decompositions can be taken from the following list:

$$
\mathfrak{V}^{0}=\mathfrak{V}_{1,+}^{0}
$$

with $\operatorname{dim} \mathfrak{V}_{1,+}^{0}=3$;

$$
\mathfrak{V}^{1}=\mathfrak{V}_{+}^{1} \oplus \mathfrak{V}_{-}^{1}, \quad \mathfrak{V}_{+}^{1}=\mathfrak{V}_{0,+}^{1} \oplus \mathfrak{V}_{2,+}^{1} \quad \mathfrak{V}_{-}^{1}=\mathfrak{V}_{1,-}^{1} \oplus \mathfrak{V}_{2,-}^{1}
$$

with respective dimensions: $\operatorname{dim} \mathfrak{V}_{+}^{1}=12=2 \oplus 10$ and $\operatorname{dim} \mathfrak{V}_{-}^{1}=8=3 \oplus 5$;

$$
\mathfrak{V}^{2}=\mathfrak{V}_{+}^{2} \oplus \mathfrak{V}_{-}^{2}, \quad \mathfrak{V}_{+}^{2}=\mathfrak{V}_{1,+}^{2} \oplus \mathfrak{V}_{2,+}^{2} \oplus \mathfrak{V}_{3,+}^{2} \quad \mathfrak{V}_{-}^{2}=\mathfrak{V}_{0,-}^{2} \oplus \mathfrak{V}_{1,-}^{2} \oplus \mathfrak{V}_{2,-}^{2} \oplus \mathfrak{V}_{3,-}^{2}
$$

with respective dimensions: $\operatorname{dim} \mathfrak{V}_{+}^{2}=49=18 \oplus 10 \oplus 21$ and $\operatorname{dim} \mathfrak{V}_{-}^{2}=43=2 \oplus 12 \oplus$ $15 \oplus 14$.

We organize all elements of the algebra $\mathfrak{h}^{-}$in the shape of irreducible tensor variables $\mathcal{O}_{j}=\left\{\mathcal{O}_{j, m} \mid-j \leq m \leq+j\right\}$ (under the action of $O(3)$ ), e.g.

$$
\begin{gathered}
J_{1}=\left\{J_{1, m} \mid-1 \leq m \leq+1\right\} \\
\left(J_{1} \cdot J_{1}\right)_{0}=\left(J_{1}^{2}\right)_{0}=\sum_{m_{1}} \sum_{m_{2}}\left\langle 1, m_{1} ; 1, m_{2} \mid 0,0\right\rangle J_{1, m_{1}} J_{1, m_{2}} \\
\left(J_{1} \cdot J_{1}\right)_{2}=\left(J_{1}^{2}\right)_{2}=\left\{\left(J_{1}^{2}\right)_{2, m}=\sum_{m_{1}} \sum_{m_{2}}\left\langle 1, m_{1} ; 1, m_{2} \mid 2, m\right\rangle J_{1, m_{1}} J_{1, m_{2}} \mid-2 \leq m \leq+2\right\},
\end{gathered}
$$

the symbols $\left\langle j_{1}, m_{1} ; j_{2}, m_{2} \mid j, m\right\rangle$ denoting the Clebsch-Gordan coefficients with the conventions of Condon and Shortley Ref. [4].

Together with some other generators of the algebra $\mathfrak{h}^{-}$besides $J_{1}$, i.e. some other irreducible tensor variables generating the algebra $\mathfrak{h}^{-}$via multiplication and Poisson bracket operation, to wit the parity even generators $B_{0}^{(1)}$ and $T_{2}$ along with the parity odd generators $S_{1}$ and $S_{2}$, a basis of the above listed vector spaces is given in terms of the components of irreducible tensor variables by

$$
\begin{aligned}
& \mathfrak{V}_{1,+}^{0}: \quad J_{1} \\
& \mathfrak{V}_{0,+}^{1}: \quad B_{0}^{(1)},\left(J_{1}^{2}\right)_{0} \quad \mathfrak{V}_{2,+}^{1}: \quad T_{2},\left(J_{1}^{2}\right)_{2} \\
& \mathfrak{V}_{1,-}^{1}: S_{1} \quad \mathfrak{V}_{2,-}^{1}: S_{2} \\
& \mathfrak{V}_{1,+}^{2}: \quad\left\{T_{2}, T_{2}\right\}_{1},\left\{S_{2}, S_{1}\right\}_{1},\left\{S_{1}, S_{1}\right\}_{1},\left(J_{1} \cdot B_{0}^{(1)}\right)_{1},\left(J_{1} \cdot T_{2}\right)_{1},\left(J_{1} \cdot\left(J_{1}^{2}\right)_{0}\right)_{1} \\
& \mathfrak{V}_{2,+}^{2}: \quad\left\{S_{2}, S_{1}\right\}_{2},\left(J_{1} \cdot T_{2}\right)_{2} \\
& \mathfrak{V}_{3,+}^{2}: \quad\left\{S_{2}, S_{1}\right\}_{3},\left(J_{1} \cdot T_{2}\right)_{3},\left(J_{1} \cdot\left(J_{1}^{2}\right)_{2}\right)_{3}
\end{aligned}
$$

and, finally,

$$
\begin{array}{ll}
\mathfrak{V}_{0,-}^{2}: & \left\{T_{2}, S_{2}\right\}_{0},\left(J_{1} \cdot S_{1}\right)_{0} \\
\mathfrak{V}_{1,-}^{2}: & \left\{T_{2}, S_{2}\right\}_{1},\left\{T_{2}, S_{1}\right\}_{1},\left(J_{1} \cdot S_{2}\right)_{1},\left(J_{1} \cdot S_{1}\right)_{1} \\
\mathfrak{V}_{2,-}^{2}: & \left\{T_{2}, S_{1}\right\}_{2},\left(J_{1} \cdot S_{2}\right)_{2},\left(J_{1} \cdot S_{1}\right)_{2} \\
\mathfrak{V}_{3,-}^{2}: & \left\{T_{2}, S_{1}\right\}_{3},\left(J_{1} \cdot S_{2}\right)_{3} .
\end{array}
$$


Here the symbols $\left(\mathcal{O}_{j_{1}} \cdot \mathcal{O}_{j_{2}}\right)_{j}$ and $\left\{\mathcal{O}_{j_{1}}, \mathcal{O}_{j_{2}}\right\}_{j}$ stand for the irreducible tensor variables $\left\{\left(\mathcal{O}_{j_{1}} \cdot \mathcal{O}_{j_{2}}\right)_{j, m} \mid-j \leq m \leq+j\right\}$ and $\left\{\left\{\mathcal{O}_{j_{1}}, \mathcal{O}_{j_{2}}\right\}_{j, m} \mid-j \leq m \leq+j\right\}$, with

$$
\left(\mathcal{O}_{j_{1}} \cdot \mathcal{O}_{j_{2}}\right)_{j, m}=\sum_{m_{1}} \sum_{m_{2}}\left\langle j_{1}, m_{1} ; j_{2}, m_{2} \mid j, m\right\rangle \mathcal{O}_{j_{1}, m_{1}} \cdot \mathcal{O}_{j_{2}, m_{2}}
$$

and

$$
\left\{\mathcal{O}_{j_{1}}, \mathcal{O}_{j_{2}}\right\}_{j, m}=\sum_{m_{1}} \sum_{m_{2}}\left\langle j_{1}, m_{1} ; j_{2}, m_{2} \mid j, m\right\rangle\left\{\mathcal{O}_{j_{1}, m_{1}}, \mathcal{O}_{j_{2}, m_{2}}\right\}
$$

respectively.

The remaining non-trivial Poisson brackets between the generators $B_{0}^{(1)}, S_{1}, S_{2}$ and $T_{2}$, i.e. the ones not listed as basis elements of $\mathfrak{V}_{+}^{2}$ and $\mathfrak{V}_{-}^{2}$, are given by

$$
\begin{aligned}
\left\{T_{2}, T_{2}\right\}_{3}= & -i\left\{S_{2}, S_{1}\right\}_{3}+i 16\left(J_{1} \cdot\left(J_{1}^{2}\right)_{2}\right)_{3}, \\
\left\{S_{2}, S_{2}\right\}_{3}= & i 2\left\{S_{2}, S_{1}\right\}_{3}-i 8\left(J_{1} \cdot T_{2}\right)_{3}-i 48\left(J_{1} \cdot\left(J_{1}^{2}\right)_{2}\right)_{3}, \\
\left\{S_{2}, S_{2}\right\}_{1}= & -i \sqrt{\frac{2}{3}}\left\{S_{2}, S_{1}\right\}_{1}-\frac{1}{6} \sqrt{5}\left\{S_{1}, S_{1}\right\}_{1}-i 16 \sqrt{\frac{2}{3}}\left(J_{1} \cdot T_{2}\right)_{1}- \\
& i 32 \sqrt{\frac{2}{15}}\left(J_{1} \cdot\left(J_{1}^{2}\right)_{0}\right)_{1}, \\
\left\{B_{0}^{(1)}, T_{2}\right\}_{2}= & i \sqrt{6}\left\{S_{2}, S_{1}\right\}_{2} ; \\
\left\{T_{2}, S_{2}\right\}_{4}= & 0 \\
\left\{T_{2}, S_{2}\right\}_{3}= & i\left\{T_{2}, S_{1}\right\}_{3}+i 8\left(J_{1} \cdot S_{2}\right)_{3}, \\
\left\{T_{2}, S_{2}\right\}_{2}= & -\frac{i}{3} \sqrt{\frac{7}{2}}\left\{T_{2}, S_{1}\right\}_{2}-i \frac{4}{3} \sqrt{14}\left(J_{1} \cdot S_{2}\right)_{2}, \\
\left\{B_{0}^{(1)}, S_{2}\right\}_{2}= & -i 2 \sqrt{\frac{2}{3}}\left\{T_{2}, S_{1}\right\}_{2}-i 4 \sqrt{\frac{2}{3}}\left(J_{1} \cdot S_{2}\right)_{2}+12\left(J_{1} \cdot S_{1}\right)_{2}, \\
\left\{B_{0}^{(1)}, S_{1}\right\}_{1}= & -i 6 \sqrt{\frac{2}{5}}\left\{T_{2}, S_{2}\right\}_{1}+2 \sqrt{\frac{3}{5}}\left\{T_{2}, S_{1}\right\}_{1}-24 \sqrt{\frac{3}{5}}\left(J_{1} \cdot S_{2}\right)_{1}+ \\
& i 12 \sqrt{2}\left(J_{1} \cdot S_{1}\right)_{1} \cdot
\end{aligned}
$$

In the sequel these relations will be referred to as generalized Poisson commutation relations.

\section{Classical Theory Part Two}

Next, we consider two massive closed strings (of the type described before and of common positive orientation) making contact in a distinguished space-time point $x^{(0)}$ and subsequently forming a single third closed string of the same type. That is, the two tube-shaped branches (numbered [1] and [2]) merge in the space-time point $x^{(0)}$ into a single third such branch (numbered [0]). The triple of branches constitutes a "string vertex". According to the laws of impact, the energy momentum vectors of the two merging strings $P_{\mu}^{[1]}$ and $P_{\mu}^{[2]}$, respectively, when added yield the energy momentum vector $P_{\mu}^{[0]}$ of the merged string. The same goes for the infinitesimal generators of the restricted Lorentz transformations $M_{\mu \nu}: M_{\mu \nu}^{[1]}+M_{\mu \nu}^{[2]}=M_{\mu \nu}^{[0]}$. This formulation refers to a fixed Lorentzian coordinate system in the $(3+1)$-dimensional Minkowski space. Since the quantities $P_{\mu}^{[\cdot]}$ and $M_{\mu \nu}^{[\cdot]}$ do not vary while the pertinent strings sweep over their branches, these quantities can just as well be assigned to the pertinent branches rather 
than to the strings. The same goes for the infinitesimal generators of the internal symmetry transformations: $J_{1}^{[\cdot]}, B_{0}^{(1)[\cdot]}, T_{2}^{[\cdot \cdot]}, S_{2}^{[\cdot]}, S_{1}^{[\cdot \cdot]}, \ldots$ They, too, are piece-wise conserved quantities.

The infinitesimal generators of rotations in the momentum rest frame of the branch $k, k=0,1,2$, are given by $\frac{1}{i_{\mathfrak{m}^{k}}}$ times the spatial components of the corresponding Pauli-Lubanski vector

$$
\mathcal{W}^{[k] \mu}=\frac{i}{2} \epsilon^{\mu \rho \sigma \nu} M_{\rho \sigma}^{[k]} P_{\nu}^{[k]}, \quad \quad \epsilon^{0123}=+1
$$

in the rest frame of the branch $k: \mathcal{W}^{[k] j}{ }_{\mid k}, j=1,2,3\left(\mathcal{W}_{\mid k}^{[k] 0}=0\right.$; the bar followed by a branch index $k$ indicates that the respective quantity is to be evaluated in the rest frame of the branch $[\mathrm{k}]$ ).

From the law of addition of the infinitesimal generators $M_{\mu \nu}^{[\cdot]}$, all that is left as a law for the composition of the spin momenta (i.e. the angular momenta corresponding to rotations in the various rest frames) is

$$
\mathcal{W}^{[0] \mu} P_{\mu}^{[1]}=\left(\mathcal{W}^{[2] \mu}-\mathcal{W}^{[1] \mu}\right) P_{\mu}^{[0]}=\mathcal{W}^{[2] \mu} P_{\mu}^{[1]}-\mathcal{W}^{[1] \mu} P_{\mu}^{[2]}
$$

Analogously, there is only one (additive) composition law relating the basis elements $J_{1}^{[k]}$ of the three-dimensional subspaces $\mathfrak{V}^{[k] 0}=\mathfrak{V}_{+}^{[k] 0}$ of $\mathfrak{h}^{[k]-}, k=0,1,2, J_{1}^{[k]}=\left\{J_{1, m}^{[k]} \mid-1 \leq\right.$ $m \leq+1\}$ with $J_{1, m}^{[k]}$ acting like the spin momenta in $\mathfrak{h}^{[k]-}$, to wit:

$$
\left(\mathcal{A}_{1 \mid 0} \cdot P_{1 \mid 0}^{[1]}\right)_{0}=0
$$

Here $\mathcal{A}_{1 \mid 0}$ stands for the following irreducible $j=1$ tensor variable in the rest frame of the branch 0

$$
\mathcal{A}_{1 \mid 0}:=-i J_{1}^{[0]}\left(P_{0 \mid 0}^{[1]} \cdot P_{0 \mid 0}^{[2]}\right)+W_{1 \mid 0}^{[1]} P_{0 \mid 0}^{[2]}+W_{1 \mid 0}^{[2]} P_{0 \mid 0}^{[1]}
$$

with $(k=1,2 ;[\neq 1]:=[2],[\neq 2]:=[1])$

$$
W_{1 \mid 0}^{[k]}:=i\left[J_{1}^{[k]} \mathfrak{m}^{[k]}+\left(J_{1}^{[k]} \cdot P_{1 \mid k}^{[\neq k]}\right)_{0} \delta^{[k]} P_{1 \mid 0}^{[k]}\right], \quad \delta^{[k]}:=\frac{\mathfrak{m}^{[k]}}{\mathfrak{m}^{[0]}} \frac{\sqrt{3}}{P_{0 \mid 0}^{[k]}+\mathfrak{m}^{[k]}}
$$

The laws of impact and the above composition law are examples of "selection rules" for the string vertex. There are infinitely many more selection rules relating basis elements of subspaces of $\mathfrak{h}^{[k]-}$ (and $\mathfrak{h}^{[k]+}$ ) of higher degrees to each other, $k=0,1,2$. However, in general apart from the elements of the Poincaré algebra and the isomorphic Poisson algebras $\mathfrak{h}^{[k]-}, k=0,1,2$, and $\mathfrak{h}^{[k]+}, k=0,1,2$, respectively, they involve a variable which encodes the phase of "breathing" of string 1 relative to the phase of "breathing" of string 2 just before the two strings merge in the point $x^{(0)}$ to form the string 0 .

For simplicity, we focus on selection rules which do not involve that kind of variable, which do not involve elements of the algebras $\mathfrak{h}^{[k]+}, k=0,1,2$, and which relate basis elements of the subspaces $\mathfrak{V}^{[k] 1}, k=0,1,2$, and $\mathfrak{V}^{[k] 2}, k=0,1,2$, respectively. Under these restrictions our analysis (first classical, then quantal) will be exhaustive.

In his diploma thesis Ref. [3] J. Großer has identified the only two "new" (tensorial) composition laws (of the restricted type) relating basis elements of the subspaces $\mathfrak{V}^{[k] 1}$, 
$k=0,1,2$, to each other. The set of these two laws is symmetric under the following substitution which we call "formal crossing": replace the real process describing the merging of branch [1] with branch [2] to form branch [0] by the non-real process describing the merging of branch [1] with crossed branch [0] to form crossed branch [2]. Here, the crossed branch is obtained from the original branch by replacing $p_{\mu}(\tau, \sigma)$ by $-p_{\mu}(\tau, \sigma)$ and by subsequently inverting the orientation of the corresponding curves $\mathcal{C}_{\tau}^{[\cdot]}$.

Großer has presented these laws in a manifestly Lorentz covariant form. For our purposes, however, it is advantageous to evaluate them in the rest frame of the merged branch [0] and to state the dependence of the contributions from the branches [1] and $[2]$ on the elements of their respective rest frame algebras $\mathfrak{h}^{[1]-}$ and $\mathfrak{h}^{[2]-}$ explicitly. For the time being, we regard the composition laws as numerical relations which follow from continuity requirements imposed on the aforementioned monodromy matrices and from factorization properties of the latter ones.

We introduce the following notation $(k=1,2)$ :

$$
\begin{aligned}
& X_{2}^{[0]}=-i \sqrt{6}\left(S_{2}^{[0]} \cdot P_{1 \mid 0}^{[1]}\right)_{2}+\left(S_{1}^{[0]} \cdot P_{1 \mid 0}^{[1]}\right)_{2}, \\
& X_{2}^{[k]}=-i \sqrt{6}\left(S_{2}^{[k]} \cdot P_{1 \mid k}^{[\neq k]}\right)_{2}+\left(S_{1}^{[k]} \cdot P_{1 \mid k}^{[\neq k]}\right)_{2}, \\
& Z_{2}^{[0]}=-2 \sqrt{3}\left(T_{2}^{[0]} \cdot\left(P_{1 \mid 0}^{[1] 2}\right)_{0}\right)_{2}+2 \sqrt{21}\left(T_{2}^{[0]} \cdot\left(P_{1 \mid 0}^{[1] 2}\right)_{2}\right)_{2}-\left(B_{0}^{(1)[0]} \cdot\left(P_{1 \mid 0}^{[1] 2}\right)_{2}\right)_{2}, \\
& Z_{2}^{[k]}=-2 \sqrt{3}\left(T_{2}^{[k]} \cdot\left(P_{1 \mid k}^{[\neq k] 2}\right)_{0}\right)_{2}+2 \sqrt{21}\left(T_{2}^{[k]} \cdot\left(P_{1 \mid k}^{[\neq k] 2}\right)_{2}\right)_{2}-\left(B_{0}^{(1)[k]} \cdot\left(P_{1 \mid k}^{[\neq k] 2}\right)_{2}\right)_{2}, \\
& \Xi_{2 \mid 0}^{[k]}=-\frac{1}{3} Z_{2}^{[k]}-\frac{1}{3} X_{2}^{[k]} \frac{\mathfrak{m}^{[0]}}{\mathfrak{m}^{[k]}}\left(P_{0 \mid 0}^{[k]}+2 \mathfrak{m}^{[k]}\right)- \\
& \frac{1}{3} \delta^{[k]}\left[\sqrt{7}\left(X_{2}^{[k]} \cdot\left(P_{1 \mid k}^{[\neq k] 2}\right)_{2}\right)_{2}-2 \sqrt{5}\left(X_{2}^{[k]} \cdot\left(P_{1 \mid k}^{[\neq k] 2}\right)_{2}\right)_{0} \frac{\left(P_{1 \mid k}^{[\neq k] 2}\right)_{2}}{\left(P_{1 \mid k}^{[\neq k] 2}\right)_{0}}\right]- \\
& \frac{4}{3}\left(J_{1}^{[k] 2}\right)_{2}\left(\frac{\mathfrak{m}^{[0]}}{\mathfrak{m}^{[k]}}\right)^{2} \frac{\left[P_{0 \mid 0}^{[k]}+\mathfrak{m}^{[k]}\right]^{3}\left[P_{0 \mid 0}^{[k]}-\mathfrak{m}^{[k]}\right]}{\left(P_{0 \mid 0}^{[k]}\right)^{2}}- \\
& \frac{4}{\sqrt{3}}\left[\sqrt{7}\left(\left(J_{1}^{[k] 2}\right)_{2} \cdot\left(P_{1 \mid k}^{[\neq k] 2}\right)_{2}\right)_{2}+2\left(J_{1}^{[k] 2}\right)_{0}\left(P_{1 \mid k}^{[\neq k] 2}\right)_{2}\right] \frac{\left[\left(P_{0 \mid 0}^{[k]}\right)^{2}-\mathfrak{m}^{[k]} P_{0 \mid 0}^{[k]}+\left(\mathfrak{m}^{[k]}\right)^{2}\right]}{\left(P_{0 \mid 0}^{[k]}\right)^{2}}+ \\
& 4 \sqrt{3}\left[\left(J_{1}^{[k]} \cdot P_{1 \mid k}^{[\neq k]}\right)_{0}\right]^{2} \frac{\left(P_{1 \mid k}^{[\neq k] 2}\right)_{2}}{\left(P_{1 \mid k}^{[\neq k] 2}\right)_{0}} \frac{\left[P_{0 \mid 0}^{[k]}-\mathfrak{m}^{[k]}\right]^{2}}{\left(P_{0 \mid 0}^{[k]}\right)^{2}}, \\
& \Theta_{2 \mid 0}^{[k]}=Z_{2}^{[k]} P_{0 \mid 0}^{[k]}+\sqrt{3}\left[-X_{2}^{[k]}\left(P_{1 \mid k}^{[\neq k] 2}\right)_{0}+\sqrt{7}\left(X_{2}^{[k]} \cdot\left(P_{1 \mid k}^{[\neq k] 2}\right)_{2}\right)_{2}-\right. \\
& \left.2 \sqrt{5}\left(X_{2}^{[k]} \cdot\left(P_{1 \mid k}^{[\neq k] 2}\right)_{2}\right)_{0} \frac{\left(P_{1 \mid k}^{[\neq k] 2}\right)_{2}}{\left(P_{1 \mid k}^{[\neq k] 2}\right)_{0}}\right] \frac{\mathfrak{m}^{[k]}}{\mathfrak{m}^{[0]}}+ \\
& 4 \sqrt{3}\left[-\left(J_{1}^{[k] 2}\right)_{2}\left(P_{1 \mid k}^{[\neq k] 2}\right)_{0}+\sqrt{7}\left(\left(J_{1}^{[k] 2}\right)_{2} \cdot\left(P_{1 \mid k}^{[\neq k] 2}\right)_{2}\right)_{2}+2\left(J_{1}^{[k] 2}\right)_{0}\left(P_{1 \mid k}^{[\neq k] 2}\right)_{2}\right] \times \\
& \frac{\left[\left(P_{0 \mid 0}^{[k]}\right)^{2}-\left(\mathfrak{m}^{[k]}\right)^{2}\right]}{P_{0 \mid 0}^{[k]}}+36\left[\left(J_{1}^{[k]} \cdot P_{1 \mid k}^{[\neq k]}\right)_{0}\right]^{2}\left(P_{1 \mid k}^{[\neq k] 2}\right)_{2} \frac{1}{P_{0 \mid 0}^{[k]}}\left(\frac{\mathfrak{m}^{[k]}}{\mathfrak{m}^{[0]}}\right)^{2}, \\
& \mathcal{B}_{1 \mid 0}=-i J_{1}^{[0]}\left(P_{0 \mid 0}^{[1]}-P_{0 \mid 0}^{[2]}\right)+W_{1 \mid 0}^{[1]}-W_{1 \mid 0}^{[2]} \text {. }
\end{aligned}
$$

Note: The irreducible tensor variables $\Xi_{2 \mid 0}^{[k]}$ and $\Theta_{2 \mid 0}^{[k]}$ are linear in $X_{2}^{[k]}$ and $Z_{2}^{[k]}$, they are quadratic in $J_{1}^{[k]}$, and they are mass and momentum dependent. 
With these abbreviations the composition laws read

$$
\begin{aligned}
X_{2}^{[0]} & =\left[\Xi_{2 \mid 0}^{[1]}-\Xi_{2 \mid 0}^{[2]}\right] \frac{1}{\mathfrak{m}^{[0]}}-4\left(\mathcal{A}_{1 \mid 0}^{2}\right)_{2} \frac{\left(P_{0 \mid 0}^{[1]}-P_{0 \mid 0}^{[2]}\right)}{\left(P_{0 \mid 0}^{[1]} \cdot P_{0 \mid 0}^{[2]}\right)^{2}}+8\left(\mathcal{A}_{1 \mid 0} \cdot \mathcal{B}_{1 \mid 0}\right)_{2} \frac{1}{\left(P_{0 \mid 0}^{[1]} \cdot P_{0 \mid 0}^{[2]}\right)}, \\
Z_{2}^{[0]} & =\left[\Theta_{2 \mid 0}^{[1]}+\Theta_{2 \mid 0}^{[2]}\right] \frac{1}{\mathfrak{m}^{[0]}}+12\left(\mathcal{A}_{1 \mid 0}^{2}\right)_{2} \frac{1}{\left(P_{0 \mid 0}^{[1]} \cdot P_{0 \mid 0}^{[2]}\right)} .
\end{aligned}
$$

The left and the right hand sides of the first law are antisymmetric under the interchange of the branches [1] and [2], the left and the right hand sides of the second law are symmetric (note: $P_{1 \mid 0}^{[2]}=-P_{1 \mid 0}^{[1]}$ ).

There are no "kinematical" constraints on the irreducible tensor variable $X_{2}^{[k]}, k=$ $0,1,2$. Hence, the components of $X_{2}^{[0]}$ furnish 5 independent linear combinations of the components of the irreducible tensor variables $S_{2}^{[0]} \in \mathfrak{V}_{-}^{[0] 1}$ and $S_{1}^{[0]} \in \mathfrak{V}_{-}^{[0] 1}$ which - with the help of $J_{1}^{[0]} \in \mathfrak{V}_{+}^{[0] 0}$ - can be expressed in terms of the basis elements of the subspaces $\mathfrak{V}^{[1] l}$ and $\mathfrak{V}^{[2] l}, l=0,1$.

In contrast, there do exist "kinematical" constraints on the irreducible tensor variable $Z_{2}^{[k]}, k=0,1,2$, to wit:

$$
\left(Z_{2}^{[0]} \cdot\left(P_{1 \mid 0}^{[1] 2}\right)_{2}\right)_{1}=0, \quad\left(Z_{2}^{[k]} \cdot\left(P_{1 \mid k}^{[\neq k] 2}\right)_{2}\right)_{1}=0, \quad k=1,2 .
$$

They imply, that there are only 3 independent linear combinations of the components of the irreducible tensor variables $B_{0}^{(1)[0]} \in \mathfrak{V}_{+}^{[0] 1}$ and $T_{2}^{[0]} \in \mathfrak{V}_{+}^{[0] 1}$ which - with the help of $J_{1}^{[0]} \in \mathfrak{V}_{+}^{[0] 0}-$ can be expressed in terms of the basis elements of the subspaces $\mathfrak{V}^{[1] l}$ and $\mathfrak{V}^{[2] l}, l=0,1$.

We express the basis elements $S_{1}^{[0]}$ and $S_{2}^{[0]}$ in terms of $X_{2}^{[0]}$ and $s_{1}:=2\left(S_{2}^{[0]} \cdot P_{1 \mid 0}^{[1]}\right)_{1}$, likewise the basis elements $B_{0}^{(1)[0]}$ and $T_{2}^{[0]}$ in terms of $Z_{2}^{[0]}$ and $t_{1}:=2\left(T_{2}^{[0]} \cdot P_{1 \mid 0}^{[1]}\right)_{1}$ :

$$
\begin{aligned}
& S_{1}^{[0]}=i \frac{1}{3} \sqrt{10}\left(s_{1} \cdot \frac{P_{1 \mid 0}^{[1]}}{\left(P_{1 \mid 0}^{1 \mid)_{0}}\right)_{0}}\right)_{1}+\frac{3}{\sqrt{5}}\left(X_{2}^{[0]} \cdot \frac{P_{10}^{[1]}}{\left(P_{1 \mid 0}^{112}\right)_{0}}\right)_{1}-\frac{1}{6} \sqrt{\frac{7}{3}}\left(X_{2}^{[0]} \cdot \frac{\left(P_{1 \mid 0}^{[1] 3}\right)_{3}}{\left(P_{1 \mid 0}^{[1] 2}\right)_{0}^{2}}\right)_{1}, \\
& S_{2}^{[0]} \quad=\frac{3}{2 \sqrt{5}}\left(s_{1} \cdot \frac{P_{1 \mid 0}^{[1]}}{\left(P_{1 \mid 0}^{[1] 2}\right)_{0}}\right)_{2}-\frac{1}{12} \sqrt{\frac{7}{3}}\left(s_{1} \cdot \frac{\left(P_{1 \mid 0}^{[1] 3}\right)_{3}}{\left(P_{1 \mid 0}^{[1] 2}\right)_{0}^{2}}\right)_{2}-i \frac{1}{5} \sqrt{2}\left(X_{2}^{[0]} \cdot \frac{P_{1 \mid 0}^{[1]}}{\left(P_{1 \mid 0}^{[1] 2}\right)_{0}}\right)_{2}+ \\
& i \frac{1}{6} \sqrt{\frac{7}{5}}\left(X_{2}^{[0]} \cdot \frac{\left(P_{1 \mid 0}^{[1] 3}\right)_{3}}{\left(P_{1 \mid 0}^{[1] 2}\right)_{0}^{2}}\right)_{2}, \\
& B_{0}^{(1)[0]}=\frac{\sqrt{15}}{2}\left(t_{1} \cdot \frac{P_{1 \mid 0}^{[1]}}{\left(P_{1 \mid 0}^{[1] 2}\right)_{0}}\right)_{0}-\frac{\sqrt{5}}{2}\left(Z_{2}^{[0]} \cdot \frac{\left(P_{1 \mid 0}^{[1] 2}\right)_{2}}{\left(P_{1 \mid 0}^{[1] 2}\right)_{0}^{2}}\right)_{0}, \\
& T_{2}^{[0]}=\frac{3}{2 \sqrt{5}}\left(t_{1} \cdot \frac{P_{1 \mid 0}^{[1]}}{\left(P_{1 \mid 0}^{(1] 2}\right)_{0}}\right)_{2}-\frac{1}{12} \sqrt{\frac{7}{3}}\left(t_{1} \cdot \frac{\left(P_{1 \mid 0}^{[1] 3}\right)_{3}}{\left(P_{1 \mid 0}^{[1] 2}\right)_{0}^{2}}\right)_{2}-\frac{1}{12 \sqrt{3}}\left(Z_{2}^{[0]} \cdot \frac{1}{\left(P_{1 \mid 0}^{(1] 2}\right)_{0}}\right)_{2}+ \\
& \frac{1}{24} \sqrt{\frac{7}{3}}\left(Z_{2}^{[0]} \cdot \frac{\left(P_{1 \mid 0}^{[1] 2}\right)_{2}}{\left(P_{1 \mid 0}^{[1] 2}\right)_{0}^{2}}\right)_{2} \text {. }
\end{aligned}
$$

There do not exist relations (of the restricted type) of the tensor variables $s_{1}$ and $t_{1}$ to the elements of the subspaces $\mathfrak{V}^{[k] 1}, k=1,2$.

We have searched for "new" relations among the basis elements of the subspaces $\mathfrak{V}^{[k] 2}, k=0,1,2$, relations of the restricted type, which are not merely the result of multiplication of relations already known. We found out that there are exactly 21 independent components of such composition laws. In more detail, our findings are: With the help of $J_{1}^{[0]}, 11$ (10) independent linear combinations of the basis elements 
of $\mathfrak{V}_{+}^{[0] 2}\left(\mathfrak{V}_{-}^{[0] 2}\right)$ can be expressed in terms of the basis elements of the subspaces $\mathfrak{V}^{[k] l}$, $k=1,2 ; 0 \leq l \leq 2$.

In order to present these composition laws, one need not start from scratch. They can be obtained from the previous ones ( $c f$. equations (11) and (2)) in a systematic way by Poisson bracket operations. Also, they can be analyzed by this tool.

This leads us to the second part of this section dedicated to the (Poisson) algebraic aspects of the relations under discussion.

Stated as above in terms of irreducible tensor multiplets, the covariance of the left hand sides and the right hand sides, respectively, of the composition laws (1) and (2) under rotations in the momentum rest frame of the merged branch is obvious. The rotations are generated by $\frac{1}{i \mathfrak{m}^{[0]}}$ times the spatial components $\mathcal{W}_{j \mid 0}^{[0]}$ of the Pauli-Lubanski vector in the aforementioned rest frame.

As far as the elements of the Poisson algebra $\mathfrak{h}^{[0]-}$ are concerned, the very same rotations are also generated by the (Cartesian) components of $J_{1}^{[0]}$. A priori, the Poisson action of $J_{1}^{[0]}$ on the components of $P_{1 \mid 0}^{[1]}=-P_{1 \mid 0}^{[2]}$ and on the elements of $\mathfrak{h}^{[k]-}, k=$ 1,2 , is not defined. We are free to define the Poisson action of $J_{1}^{[0]}$ on these objects provided the definition complies with the formal properties of Poisson algebras and provided it ensures that the action of $J_{1}^{[0]}$ takes valid relations into valid relations. In particular, this implies that the action of $J_{1}^{[0]}$ on all irreducible tensor variables in the rest frame of the merged branch such as $P_{1 \mid 0}^{[1]}=-P_{1 \mid 0}^{[2]}, W_{1 \mid 0}^{[k]}, k=1,2, \Xi_{2 \mid 0}^{[k]}$, $\Theta_{2 \mid 0}^{[k]}, k=1,2$, is identical with the action of $\frac{1}{i \mathfrak{m}^{[0]}} \mathcal{W}_{1 \mid 0}^{[0]}=\frac{1}{i \mathfrak{m}^{[0]}}\left(\mathcal{W}_{1,-1}^{[0]}, \mathcal{W}_{1,0}^{[0]}, \mathcal{W}_{1,+1}^{[0]}\right)=$ $\frac{1}{i \mathfrak{m}^{[0]}}\left(\frac{1}{\sqrt{2}}\left(\mathcal{W}_{1 \mid 0}^{[0]}-i \mathcal{W}_{2 \mid 0}^{[0]}\right), \mathcal{W}_{3 \mid 0}^{[0]}, \frac{-1}{\sqrt{2}}\left(\mathcal{W}_{1 \mid 0}^{[0]}+i \mathcal{W}_{2 \mid 0}^{[0]}\right)\right)$. Since the elements of $\mathfrak{h}^{[k]-}, k=0,1,2$, apart from momentum densities, encode coordinate differences only, the components of the generator $P_{\mu}^{[0]}$ of joint rigid translations of all three branches have vanishing Poisson brackets with each and every one of these elements. Thus, $P_{0 \mid 0}^{[0]}=\mathfrak{m}^{[0]}$ plays the rôle of a common central element. The components of the vectors $P_{\mu}^{[1]}$ and $P_{\mu}^{[2]}$ not only Poisson commute among each other, but they have also vanishing Poisson brackets with all elements of $\mathfrak{h}^{[k]-}, k=1,2$. The same goes for their projections $P_{1 \mid 0}^{[1]}=-P_{1 \mid 0}^{[2]}, P_{0 \mid 0}^{[1]}$ and $P_{0 \mid 0}^{[2]}$. Minkowski space-time causality implies that the elements of $\mathfrak{h}^{[1]-}$ and the elements of $\mathfrak{h}^{[2]-}$ mutually Poisson commute. The Poisson brackets of $P_{1 \mid 0}^{[1]}=-P_{1 \mid 0}^{[2]}$ and $P_{0 \mid 0}^{[1]}-P_{0 \mid 0}^{[2]}\left(P_{0 \mid 0}^{[1]}+P_{0 \mid 0}^{[2]}=\mathfrak{m}^{[0]}\right.$ !) with the generators $B_{0}^{(1)[0]}, S_{1}^{[0]}, S_{2}^{[0]}, T_{2}^{[0]}, \ldots$ of the algebra $\mathfrak{h}^{[0]-}$ are not defined a priori. In fact, their definition is a rather delicate matter. We get a first hint as to their definition from the composition laws (1) and (2) focussing on their algebraic rather than their numerical aspects. Defining the Poisson brackets $\left\{X_{2}^{[0]}, P_{1 \mid 0}^{[1]}\right\}_{L},\left\{Z_{2}^{[0]}, P_{1 \mid 0}^{[1]}\right\}_{L}, L=1,2,3,\left\{X_{2}^{[0]},\left(P_{0 \mid 0}^{[1]}-P_{0 \mid 0}^{[2]}\right)\right\}_{2}$ and $\left\{Z_{2}^{[0]},\left(P_{0 \mid 0}^{[1]}-P_{0 \mid 0}^{[2]}\right)\right\}_{2}$ in accordance with the right hand sides of the composition laws, we are led to the following specifications

$$
\begin{aligned}
\left\{X_{2}^{[0]}, P_{1 \mid 0}^{[1]}\right\}_{L}= & \begin{cases}4 \sqrt{\frac{10}{3}}\left(\mathcal{B}_{1 \mid 0} \cdot P_{1 \mid 0}^{[1]}\right)_{1} & \text { for } L=1, \\
4 \sqrt{6}\left(\mathcal{B}_{1 \mid 0} \cdot P_{1 \mid 0}^{[1]}\right)_{2} & \text { for } L=2, \\
0 & \text { for } L=3,\end{cases} \\
\left\{X_{2}^{[0]},\left(P_{0 \mid 0}^{[1]}-P_{0 \mid 0}^{[2]}\right)\right\}_{2}=0 ; &
\end{aligned}
$$




$$
\begin{aligned}
\left\{Z_{2}^{[0]}, P_{1 \mid 0}^{[1]}\right\}_{L}= & \begin{cases}4 \sqrt{30}\left(\mathcal{A}_{1 \mid 0} \cdot P_{1 \mid 0}^{[1]}\right)_{1} & \text { for } L=1, \\
12 \sqrt{6}\left(\mathcal{A}_{1 \mid 0} \cdot P_{1 \mid 0}^{[1]}\right)_{2} & \text { for } L=2, \\
0 & \text { for } L=3,\end{cases} \\
& \left\{Z_{2}^{[0]},\left(P_{0 \mid 0}^{[1]}-P_{0 \mid 0}^{[2]}\right)\right\}_{2}=0 .
\end{aligned}
$$

Next we shall test the consistency of the above specifications with the generalized Poisson commutation relations for the three branches, the three corresponding sets of relations being isomorphic to each other. As we already found out, there are exactly 10 (11) "new" composition laws in the $P$-linear span of the subspaces $\mathfrak{V}^{[k] 2}, k=0,1,2$, antisymmetric (symmetric) under the exchange of the branches [1] and [2] (for the concept of $P$ linearity and related concepts consult Ref. [5]). The Poisson bracket operation based on the assignments given so far must take valid relations into valid relations.

Naively, i.e. not paying attention to the generalized Poisson commutation relations, the 5 (3) independent components of the composition laws $X_{2}^{[0]}=\ldots\left(Z_{2}^{[0]}=\ldots\right)$ by Poisson bracket operations should produce 15 (13) valid "new" composition laws of the antisymmetric and symmetric type, respectively. Hence, the components of the composition laws so produced are subject to at least 5 (2) constraints due to the generalized Poisson commutation relations.

For reasons of manifest $O(3)$-invariance, we do not solve the kinematical constraint on the composition law (2) for the independent corresponding components of the left hand side $Z_{2}^{[0]}$ and the right hand side, respectively. Instead, we list the $P$-linearly independent combinations of the Poisson brackets $\left\{Z_{2}^{[0]}, X_{2}^{[0]}\right\}_{L} ;\left\{X_{2}^{[0]}, X_{2}^{[0]}\right\}_{L}$ and $\left\{Z_{2}^{[0]}, Z_{2}^{[0]}\right\}_{L}$ which are not reduced to $P$-linear combinations of the products $\left(Z_{2}^{[0]} \cdot \mathcal{B}_{1 \mid 0}\right)_{j},\left(X_{2}^{[0]} \cdot \mathcal{A}_{1 \mid 0}\right)_{j}$, $\left(Z_{2}^{[0]} \cdot \mathcal{A}_{1 \mid 0}\right)_{j}$ and $\left(X_{2}^{[0]} \cdot \mathcal{B}_{1 \mid 0}\right)_{j}$ by the kinematical constraints. We begin with the 15 combinations involving the Poisson brackets which are antisymmetric under the interchange of the merging branches. They are identified as the components of $\left\{Z_{2}^{[0]}, X_{2}^{[0]}\right\}_{L}, L=0,2,3$ and the two $P$-linearly independent combinations of the components of the irreducible tensor $\left\{Z_{2}^{[0]}, X_{2}^{[0]}\right\}_{4}$ which are not contained in the $P$-linear span of the components of the irreducible tensor $\left(\left\{Z_{2}^{[0]}, X_{2}^{[0]}\right\}_{4} \cdot P_{1 \mid 0}^{[1]}\right)_{3}$.

Now we turn to the 13 combinations involving the Poisson brackets which are symmetric under the interchange of the merging branches. They are identified as the components of $\left\{X_{2}^{[0]}, X_{2}^{[0]}\right\}_{L}, L=1,3$, the "projection" $\left(\left\{Z_{2}^{[0]}, Z_{2}^{[0]}\right\}_{3} \cdot\left(P_{1 \mid 0}^{[1] 3}\right)_{3}\right)_{0}$, and the two $P$-linearly independent combinations of the components of the irreducible tensor $\mathbb{Z}_{2}:=\left(\left\{Z_{2}^{[0]}, Z_{2}^{[0]}\right\}_{3} \cdot\left(P_{1 \mid 0}^{[1] 2}\right)_{2}\right)_{2}$ which are not contained in the $P$-linear span of the components of the irreducible tensor $\left(\mathbb{Z}_{2} \cdot P_{1 \mid 0}^{[1]}\right)_{1}$.

Apart from the formal properties of the Poisson brackets, i.e. antisymmetry, Jacobi identity and Leibniz rule, the only concretely specified mixed Poisson brackets used for the transport of the kinematical constraints on the components of $Z_{2}^{[0]}$ to those on the Poisson brackets of the components of $X_{2}^{[0]}$ and $Z_{2}^{[0]}$ are the ones given above, viz. $\left\{X_{2}^{[0]}, P_{1 \mid 0}^{[1]}\right\}_{L},\left\{Z_{2}^{[0]}, P_{1 \mid 0}^{[1]}\right\}_{L}$ and $\left\{J_{1}^{[0]}, \mathcal{O}_{j}^{[k]}\right\}_{L}, k=1,2$. These concrete specifications were designed to be compatible with the composition laws $X_{2}^{[0]}=\ldots, Z_{2}^{[0]}=\ldots$ Thus the kinematical constraints on the components of $\left\{Z_{2}^{[0]}, X_{2}^{[0]}\right\}_{L}$ and $\left\{Z_{2}^{[0]}, Z_{2}^{[0]}\right\}_{L}$ are automatically satisfied if for $X_{2}^{[0]}$ and $Z_{2}^{[0]}$ the right hand sides of their composition laws are inserted. 
Next we exhibit the additional "dynamical" constraints (due to the generalized Poisson commutation relations for the merged branch) on the $15+13 P$-linear combinations of the Poisson brackets $\left\{Z_{2}^{[0]}, X_{2}^{[0]}\right\}_{L} ;\left\{X_{2}^{[0]}, X_{2}^{[0]}\right\}_{L}$ and $\left\{Z_{2}^{[0]}, Z_{2}^{[0]}\right\}_{L}$ listed above.

$$
\begin{aligned}
& 0=i\left(\left\{Z_{2}^{[0]}, X_{2}^{[0]}\right\}_{2} \cdot\left(P_{1 \mid 0}^{[1] 2}\right)_{2}\right)_{0}+i \frac{10}{7 \sqrt{7}}\left(\left\{Z_{2}^{[0]}, X_{2}^{[0]}\right\}_{0} \cdot\left(P_{1 \mid 0}^{[1] 2}\right)_{0}\right)_{0}+ \\
& \frac{192}{7} \sqrt{\frac{6}{7}}\left(\left(X_{2}^{[0]} \cdot J_{1}^{[0]}\right)_{2} \cdot\left(P_{1 \mid 0}^{[1] 2}\right)_{0}\left(P_{1 \mid 0}^{[1] 2}\right)_{2}\right)_{0}+i \frac{192}{7} \sqrt{\frac{2}{7}}\left(\left(X_{2}^{[0]} \cdot \mathcal{A}_{1 \mid 0}\right)_{2} \cdot\left(P_{1 \mid 0}^{[1] 2}\right)_{2}\right)_{0}, \\
& 0=i\left(\left\{Z_{2}^{[0]}, X_{2}^{[0]}\right\}_{3} \cdot\left(P_{1 \mid 0}^{[1] 2}\right)_{0}\left(P_{1 \mid 0}^{[1] 2}\right)_{2}\right)_{2}-i \sqrt{\frac{7}{10}}\left(\left\{Z_{2}^{[0]}, X_{2}^{[0]}\right\}_{3} \cdot\left(P_{1 \mid 0}^{[1] 4}\right)_{4}\right)_{2}+ \\
& 16 \sqrt{3}\left(\left(X_{2}^{[0]} \cdot J_{1}^{[0]}\right)_{3} \cdot\left(P_{1 \mid 0}^{[1] 2}\right)_{0}{ }^{2}\left(P_{1 \mid 0}^{[1] 2}\right)_{2}\right)_{2}-8 \sqrt{\frac{42}{5}}\left(\left(X_{2}^{[0]} \cdot J_{1}^{[0]}\right)_{3} \cdot\left(P_{1 \mid 0}^{[1] 2}\right)_{0}\left(P_{1 \mid 0}^{[1] 4}\right)_{4}\right)_{2}- \\
& \frac{16}{5} \sqrt{\frac{42}{5}}\left(\left(X_{2}^{[0]} \cdot J_{1}^{[0]}\right)_{2} \cdot\left(P_{1 \mid 0}^{[1] 2}\right)_{0}^{3}\right)_{2}+16 \sqrt{\frac{6}{5}}\left(\left(X_{2}^{[0]} \cdot J_{1}^{[0]}\right)_{2} \cdot\left(P_{1 \mid 0}^{[1] 2}\right)_{0}{ }^{2}\left(P_{1 \mid 0}^{[1] 2}\right)_{2}\right)_{2}- \\
& \frac{4}{5} \sqrt{42}\left(\left(X_{2}^{[0]} \cdot J_{1}^{[0]}\right)_{2} \cdot\left(P_{1 \mid 0}^{[1] 2}\right)_{0}\left(P_{1 \mid 0}^{[1] 4}\right)_{4}\right)_{2}+i \frac{48}{5} \sqrt{\frac{14}{5}}\left(\left(X_{2}^{[0]} \cdot \mathcal{A}_{1 \mid 0}\right)_{2} \cdot\left(P_{1 \mid 0}^{[1] 2}\right)_{0}^{2}\right)_{2}- \\
& i 48 \sqrt{\frac{2}{5}}\left(\left(X_{2}^{[0]} \cdot \mathcal{A}_{1 \mid 0}\right)_{2} \cdot\left(P_{1 \mid 0}^{[1] 2}\right)_{0}\left(P_{1 \mid 0}^{[1] 2}\right)_{2}\right)_{2}+i \frac{12}{5} \sqrt{14}\left(\left(X_{2}^{[0]} \cdot \mathcal{A}_{1 \mid 0}\right)_{2} \cdot\left(P_{1 \mid 0}^{[1] 4}\right)_{4}\right)_{2}, \\
& 0=-i \frac{126}{\sqrt{5}}\left(\left\{Z_{2}^{[0]}, X_{2}^{[0]}\right\}_{4} \cdot\left(P_{1 \mid 0}^{[1] 2}\right)_{0}^{2}\right)_{4}+i 78 \sqrt{2}\left(\left\{Z_{2}^{[0]}, X_{2}^{[0]}\right\}_{3} \cdot\left(P_{1 \mid 0}^{[1] 2}\right)_{0}\left(P_{1 \mid 0}^{[1] 2}\right)_{2}\right)_{4}+ \\
& i 12 \sqrt{385}\left(\left\{Z_{2}^{[0]}, X_{2}^{[0]}\right\}_{3} \cdot\left(P_{1 \mid 0}^{[1] 4}\right)_{4}\right)_{4}-i 153 \sqrt{\frac{7}{5}}\left(\left\{Z_{2}^{[0]}, X_{2}^{[0]}\right\}_{2} \cdot\left(P_{1 \mid 0}^{[1] 2}\right)_{0}\left(P_{1 \mid 0}^{[1] 2}\right)_{2}\right)_{4}- \\
& i 35 \sqrt{\frac{11}{2}}\left(\left\{Z_{2}^{[0]}, X_{2}^{[0]}\right\}_{2} \cdot\left(P_{1 \mid 0}^{[1] 4}\right)_{4}\right)_{4}-i 127\left(\left\{Z_{2}^{[0]}, X_{2}^{[0]}\right\}_{0} \cdot\left(P_{1 \mid 0}^{[1] 4}\right)_{4}\right)_{4}- \\
& i \frac{994}{3} \sqrt{2}\left(\left(Z_{2}^{[0]} \cdot \mathcal{B}_{1 \mid 0}\right)_{3} \cdot\left(P_{1 \mid 0}^{[1] 2}\right)_{0}\left(P_{1 \mid 0}^{[1] 2}\right)_{2}\right)_{4}-i \frac{301}{3} \sqrt{385}\left(\left(Z_{2}^{[0]} \cdot \mathcal{B}_{1 \mid 0}\right)_{3} \cdot\left(P_{1 \mid 0}^{[1] 4}\right)_{4}\right)_{4}+ \\
& i \frac{17906}{3} \sqrt{\frac{2}{5}}\left(\left(Z_{2}^{[0]} \cdot \mathcal{B}_{1 \mid 0}\right)_{2} \cdot\left(P_{1 \mid 0}^{[1] 2}\right)_{0}\left(P_{1 \mid 0}^{[1] 2}\right)_{2}\right)_{4}+i \frac{679}{6} \sqrt{77}\left(\left(Z_{2}^{[0]} \cdot \mathcal{B}_{1 \mid 0}\right)_{2} \cdot\left(P_{1 \mid 0}^{[1] 4}\right)_{4}\right)_{4}+ \\
& i \frac{1925}{2} \sqrt{\frac{5}{3}}\left(\left(Z_{2}^{[0]} \cdot \mathcal{B}_{1 \mid 0}\right)_{1} \cdot\left(P_{1 \mid 0}^{[1] 4}\right)_{4}\right)_{4}+1024 \sqrt{6}\left(\left(X_{2}^{[0]} \cdot J_{1}^{[0]}\right)_{3} \cdot\left(P_{1 \mid 0}^{[1] 2}\right)_{0}{ }^{2}\left(P_{1 \mid 0}^{[1] 2}\right)_{2}\right)_{4}+ \\
& 768 \sqrt{\frac{105}{11}}\left(\left(X_{2}^{[0]} \cdot J_{1}^{[0]}\right)_{3} \cdot\left(P_{1 \mid 0}^{[1] 2}\right)_{0}\left(P_{1 \mid 0}^{[1] 4}\right)_{4}\right)_{4}-1792 \sqrt{\frac{91}{15}}\left(\left(X_{2}^{[0]} \cdot J_{1}^{[0]}\right)_{3} \cdot\left(P_{1 \mid 0}^{[1] 6}\right)_{6}\right)_{4}- \\
& \frac{21536}{7} \sqrt{\frac{6}{5}}\left(\left(X_{2}^{[0]} \cdot J_{1}^{[0]}\right)_{2} \cdot\left(P_{1 \mid 0}^{[1] 2}\right)_{0}{ }^{2}\left(P_{1 \mid 0}^{[1] 2}\right)_{2}\right)_{4}+ \\
& 3552 \sqrt{\frac{3}{77}}\left(\left(X_{2}^{[0]} \cdot J_{1}^{[0]}\right)_{2} \cdot\left(P_{1 \mid 0}^{[1] 2}\right)_{0}\left(P_{1 \mid 0}^{[1] 4}\right)_{4}\right)_{4}- \\
& 808 \sqrt{\frac{130}{3}}\left(\left(X_{2}^{[0]} \cdot J_{1}^{[0]}\right)_{2} \cdot\left(P_{1 \mid 0}^{[1] 6}\right)_{6}\right)_{4}+i \frac{14304}{7} \sqrt{\frac{2}{5}}\left(\left(X_{2}^{[0]} \cdot \mathcal{A}_{1 \mid 0}\right)_{2} \cdot\left(P_{1 \mid 0}^{[1] 2}\right)_{0}\left(P_{1 \mid 0}^{[1] 2}\right)_{2}\right)_{4}- \\
& i \frac{60960}{\sqrt{77}}\left(\left(X_{2}^{[0]} \cdot \mathcal{A}_{1 \mid 0}\right)_{2} \cdot\left(P_{1 \mid 0}^{[1] 4}\right)_{4}\right)_{4}-i 152 \sqrt{\frac{26}{5}}\left(\left(X_{2}^{[0]} \cdot \mathcal{A}_{1 \mid 0}\right)_{2} \cdot \frac{\left(P_{1 \mid 0}^{[1] 6}\right)_{6}}{\left(P_{1 \mid 0}^{[1] 2}\right)_{0}}\right)_{4} ;
\end{aligned}
$$




$$
\begin{aligned}
0= & i \frac{1}{\sqrt{3}}\left(\left\{Z_{2}^{[0]}, Z_{2}^{[0]}\right\}_{3} \cdot\left(P_{1 \mid 0}^{[1] 2}\right)_{2}\right)_{2}-i \frac{45}{7}\left(\left\{X_{2}^{[0]}, X_{2}^{[0]}\right\}_{3} \cdot\left(P_{1 \mid 0}^{[1] 2}\right)_{0}\left(P_{1 \mid 0}^{[1] 2}\right)_{2}\right)_{2}+ \\
& i 9 \sqrt{\frac{5}{14}}\left(\left\{X_{2}^{[0]}, X_{2}^{[0]}\right\}_{3} \cdot\left(P_{1 \mid 0}^{[1] 4}\right)_{4}\right)_{2}+i 52 \sqrt{\frac{6}{35}}\left(\left(Z_{2}^{[0]} \cdot \mathcal{A}_{1 \mid 0}\right)_{2} \cdot\left(P_{1 \mid 0}^{[1] 2}\right)_{0}\right)_{2}+ \\
& i 12 \sqrt{\frac{6}{7}}\left(\left(Z_{2}^{[0]} \cdot \mathcal{A}_{1 \mid 0}\right)_{1} \cdot\left(P_{1 \mid 0}^{[1] 2}\right)_{2}\right)_{2}+i \frac{480}{7}\left(\left(X_{2}^{[0]} \cdot \mathcal{B}_{1 \mid 0}\right)_{3} \cdot\left(P_{1 \mid 0}^{[1] 2}\right)_{0}\left(P_{1 \mid 0}^{[1] 2}\right)_{2}\right)_{2}- \\
& i 48 \sqrt{\frac{10}{7}}\left(\left(X_{2}^{[0]} \cdot \mathcal{B}_{1 \mid 0}\right)_{3} \cdot\left(P_{1 \mid 0}^{[1] 4}\right)_{4}\right)_{2}-i 96 \sqrt{\frac{2}{35}}\left(\left(X_{2}^{[0]} \cdot \mathcal{B}_{1 \mid 0}\right)_{2} \cdot\left(P_{1 \mid 0}^{[1] 2}\right)_{0}\right)_{2}+ \\
& i \frac{96}{7} \sqrt{10}\left(\left(X_{2}^{[0]} \cdot \mathcal{B}_{1 \mid 0}\right)_{2} \cdot\left(P_{1 \mid 0}^{[1] 2}\right)_{0}\left(P_{1 \mid 0}^{[1] 2}\right)_{2}\right)_{2}-i 24 \sqrt{\frac{2}{7}}\left(\left(X_{2}^{[0]} \cdot \mathcal{B}_{1 \mid 0}\right)_{2} \cdot\left(P_{1 \mid 0}^{[1] 4}\right)_{4}\right)_{2}- \\
& \frac{960}{7} \sqrt{3}\left(\left(\left(J_{1}^{[0]} \cdot J_{1}^{[0]}\right)_{2} \cdot J_{1}^{[0]}\right)_{3} \cdot\left(P_{1 \mid 0}^{[1] 2}\right)_{0}{ }^{2}\left(P_{1 \mid 0}^{[1] 2}\right)_{2}\right)_{2}+ \\
& 96 \sqrt{\frac{30}{7}}\left(\left(\left(J_{1}^{[0]} \cdot J_{1}^{[0]}\right)_{2} \cdot J_{1}^{[0]}\right)_{3} \cdot\left(P_{1 \mid 0}^{[1] 2}\right)_{0}\left(P_{1 \mid 0}^{[1] 4}\right)_{4}\right)_{2} \cdot
\end{aligned}
$$

In the sequel we shall refer to the right hand sides of the preceeding constraint equations as DYN1, DYN2, DYN3, and DYN0 respectively.

10 [!] $P$-linear independent combinations of the components of the Poisson brackets $\left\{Z_{2}^{[0]}, X_{2}^{[0]}\right\}_{L}, L=2,3$, remain unconstrained. A convenient choice of these combinations consists in the 5 components of the irreducible tensor $\left\{Z_{2}^{[0]}, X_{2}^{[0]}\right\}_{2}$ and $5 P$-linearly independent combinations of the components of the irreducible tensor $\left\{Z_{2}^{[0]}, X_{2}^{[0]}\right\}_{3}$ which are not constrained by DYN2 $=0$.

11 [!] P-linear independent combinations of the components of the Poisson brackets $\left\{X_{2}^{[0]}, X_{2}^{[0]}\right\}_{L}$ and $\left\{Z_{2}^{[0]}, Z_{2}^{[0]}\right\}_{L}$ remain unconstrained. A convenient choice of these combinations consists in the $3+7$ components of the irreducible tensors $\left\{X_{2}^{[0]}, X_{2}^{[0]}\right\}_{L}$, $L=1,3$ and the "projection" $\left(\left\{Z_{2}^{[0]}, Z_{2}^{[0]}\right\}_{3} \cdot\left(P_{1 \mid 0}^{[1] 3}\right)_{3}\right)_{0}$.

The derivation of the above dynamical constraints does not require any specifications of the "mixed" Poisson brackets beyond the ones already stated in the context of the kinematical constraints. If for $X_{2}^{[0]}$ and $Z_{2}^{[0]}$ the right hand sides of their composition laws are inserted and the (isomorphic) generalized Poisson commutation relations for the merging branches are used, the dynamical constraints are identically satisfied. Thus, the concrete specifications for the "mixed" Poisson brackets, given so far, pass the test of consistency with the generalized Poisson commutation relations for the three branches.

Comparing the numbers for the independent combinations of the components $\left\{Z_{2}^{[0]}, X_{2}^{[0]}\right\}_{L, M} ;\left\{X_{2}^{[0]}, X_{2}^{[0]}\right\}_{L, M}$ and $\left\{Z_{2}^{[0]}, Z_{2}^{[0]}\right\}_{L, M}$ which remain unconstrained, on the one hand, with the previously determined numbers for the independent new relations (of the restricted type) among the basis elements of the subspaces $\mathfrak{V}^{[k] l}, k=0,1,2$, $0 \leq l \leq 2$, on the other hand, we conclude that the latter relations can all be obtained from the composition laws $X_{2}^{[0]}=\ldots$ and $Z_{2}^{[0]}=\ldots$ by Poisson bracket induction. Moreover, produced in this way they are automatically organized in the irreducible tensor multiplets:

$$
\begin{aligned}
& \left\{Z_{2}^{[0]}, X_{2}^{[0]}\right\}_{2}=\ldots, \\
& \left\{Z_{2}^{[0]}, X_{2}^{[0]}\right\}_{3} /_{\text {DYN2 }=0}=\ldots \ldots \ldots /_{\text {DYN2RHS }=0} ; \\
& \left\{X_{2}^{[0]}, X_{2}^{[0]}\right\}_{L}=\ldots, \quad L=1,3 \\
& \left(\left\{Z_{2}^{[0]}, Z_{2}^{[0]}\right\}_{3} \cdot\left(P_{1 \mid 0}^{[1] 3}\right)_{3}\right)_{0}=\ldots
\end{aligned}
$$


Here the dots and the symbol DYN2RHS denote the result of inserting the right hand sides of the composition laws $X_{2}^{[0]}=\ldots$ and $Z_{2}^{[0]}=\ldots$ into the Poisson brackets and into the irreducible tensor DYN2, respectively, on the pertinent left hand sides.

Just for the record: these manifestly covariant "new" composition laws are new only as far as they cannot be produced by multiplication from relations among the basis elements of the subspaces $\mathfrak{V}^{[k]}, k=0,1,2,0 \leq l \leq 1$.

Before we turn to the quantization of the composition laws $X_{2}^{[0]}=\ldots$ and $Z_{2}^{[0]}=\ldots$, we note in passing that the explicit expressions for the generators $T_{2}^{[0]}$ and $S_{2}^{[0]}$ in terms of matrix elements of the relevant monodromy matrix suggest the following specifications for the Poisson brackets
(i) $\left\{T_{2}^{[0]}, P_{1 \mid 0}^{[1]}\right\}_{2}=0$
(ii) $\left\{T_{2}^{[0]}, P_{1 \mid 0}^{[1]}\right\}_{3}=0$
and
(iii) $\left\{S_{2}^{[0]}, P_{1 \mid 0}^{[1]}\right\}_{3}=0$.

This observation is based on the fact that the first entries $T_{2, m_{1}}^{[0]}\left(S_{2, m_{1}}^{[0]}\right)$ in the Poisson brackets of the non-vanishing contributions

$$
\left\langle 2, m_{1} ; 1, m_{2} \mid L, M\right\rangle\left\{T_{2, m_{1}}^{[0]}, P_{1, m_{2} \mid 0}^{[1]}\right\} \quad\left(\left\langle 2, m_{1} ; 1, m_{2} \mid L, M\right\rangle\left\{S_{2, m_{1}}^{[0]}, P_{1, m_{2} \mid 0}^{[1]}\right\}\right)
$$

to the left hand sides of $(i) \&$ (ii) and of $(i i i)$, respectively, do not contain dynamical variables which are canonically conjugate to the dynamical variables contained in the corresponding second entries $P_{1, m_{2} \mid 0^{[1]}}$.

For a similar reason we expect that an evaluation of the Poisson brackets of $S_{2}^{[0]}$ and $S_{1}^{[0]}$ with $\left(P_{0 \mid 0}^{[1]}-P_{0 \mid 0}^{[2]}\right)$ would result in $P$-linear combinations of the components $J_{1, m}^{[0]}$.

The importance of these observations for the quantization of the composition laws (11) and (2) will become clear in the next section.

\section{Quantum Theory}

In this section we explore the chances that the previously presented classical composition laws can be quantized and we narrow down the shape they would take in the quantum theory.

To shed light on these issues, we stick to the strategy of Ref. [1] :

We make use of the 1:1 correspondence established there between the classical observables $J_{1}^{[k]}, B_{0}^{(1)[k]}, S_{1}^{[k]}, S_{2}^{[k]}, T_{2}^{[k]}, \ldots$ on the one hand, and the quantum observables

$$
\begin{array}{ccc}
\hat{J}_{1}^{[0]}=\frac{\hbar}{2 \pi \alpha^{\prime}} \hat{X}_{1}^{[k]}, & \hat{B}_{0}^{(1)[0]}=\left(\frac{\hbar}{2 \pi \alpha^{\prime}}\right)^{2} \hat{\mathbb{B}}_{0}^{(1)[k]}, \quad \hat{S}_{2}^{[0]}=\left(\frac{\hbar}{2 \pi \alpha^{\prime}}\right)^{2} \hat{\mathbb{S}}_{1}^{[k]}, \\
\hat{S}_{1}^{[0]}=\left(\frac{\hbar}{2 \pi \alpha^{\prime}}\right)^{2} \hat{\mathbb{S}}_{2}^{[k]}, & \hat{T}_{2}^{[0]}=\left(\frac{\hbar}{2 \pi \alpha^{\prime}}\right)^{2} \hat{\mathscr{X}}_{2}^{[k]}, \quad \ldots \quad & k=0,1,2
\end{array}
$$

on the other hand.

Here for each separate value of $k$, the "slashed" quantities act as hermitian, scale invariant generators of quantum $*$-algebras $\hat{\mathfrak{h}}^{[k]-}$ which correspond to the classical $*-$ algebras $\mathfrak{h}^{[k]-}, k=0,1,2$. The validity of the generalized commutation relations for each 
branch separately is left untouched. The elements of the algebras $\hat{\mathfrak{h}}^{[1]-}$ and $\hat{\mathfrak{h}}^{[2]-}$ commute with one another for reasons of causality as before. The quantum counterparts $\hat{P}_{\mu}^{[k]}$ of the components of the energy momentum vectors $P_{\mu}^{[k]}$ act as central elements for each algebra $\mathfrak{h}^{[k]-}, k=0,1,2$, separately. Moreover, the quantum counterparts $\hat{P}_{\mu}^{[0]}$ act as global central elements for all three algebras $\mathfrak{h}^{[k]-}, k=0,1,2$, and beyond for all quantum observables of the string vertex built from the right-movers. Hence, without loss of generality, the components $\hat{P}_{\mu}^{[0]}$, in particular the (total mass) ${ }^{2}$-operator $\left(\hat{P}^{[0]}\right)^{2}=\hat{P}_{\mu}^{[0]} \hat{P}^{[0] \mu}$, may be treated as $c$-numbers and may be fixed. Since we are primarily interested in vertices describing real processes with a positive mass $\mathfrak{m}^{[0]}$ for the merged branch, we fix the components of $\hat{P}_{\mu}^{[0]}, \mu=0,1,2,3$, as numbers in the forward light-cone in momentum space. Due to Lorentz invariance, we may even set

$$
\hat{P}_{\mu}^{[0]}=\delta_{\mu}^{0} \mathfrak{m}^{[0]}, \quad \mathfrak{m}^{[0]}>0 .
$$

This defines the (class of) rest frame(s) for the merged branch. Energy-momentum conservation requires the following relations for the branch [0] rest frame components $\hat{P}_{0 \mid 0}^{[k]}:=\left(\hat{P}_{\mu}^{[k]} \hat{P}^{[0] \mu}\right) / \mathfrak{m}^{[0]}$ and $\hat{P}_{n \mid 0}^{[k]}, k=1,2: \hat{P}_{0 \mid 0}^{[1]}+\hat{P}_{0 \mid 0}^{[2]}=\mathfrak{m}^{[0]}$ and $\hat{P}_{n \mid 0}^{[1]}+\hat{P}_{n \mid 0}^{[2]}=0$, $n=1,2,3$, or rather $\hat{P}_{1 \mid 0}^{[1]}=-\hat{P}_{1 \mid 0}^{[2]}$ referring to a branch [0] rest frame spin basis.

The components of the three operator-valued vectors $\hat{P}_{\mu}^{[k]}, \mu=0,1,2,3$, can be simultaneously diagonalized. Hence, for the real vertex processes we have in mind, it is legitimate to limit our angle of sight of the entire algebra to the perspective from its momentum type representations, for which $\hat{P}_{1, m \mid 0}^{[1]}, m=-1,0,+1$ and $\left(\hat{P}_{0 \mid 0}^{[1]}-\hat{P}_{0 \mid 0}^{[2]}\right)$ act as multiplication operators. Thus we take the liberty of dropping the hats from the $\hat{P}_{\text {.. }}^{[\cdot]}$ operators, at the same same time paying utmost attention to the order in which the latter ones appear when combined with non-commuting partners.

We postulate the validity of the following "mixed" commutation relations

$$
\left[\hat{X}_{1}^{[0]}, \hat{\mathcal{O}}_{j}\right]_{L}=-\delta_{L, j} \sqrt{j(j+1)} \hat{\mathcal{O}}_{j}, \quad j=0,1,2, \ldots
$$

for all irreducible tensor operators $\hat{\mathcal{O}}_{j}$ contained in $\hat{\mathfrak{h}}^{[k]}, k=0,1,2$, in particular:

$$
\left[\hat{X}_{1}^{[0]}, P_{1 \mid 0}^{[1]}\right]_{L}=-\delta_{L, 1} \sqrt{2} P_{1 \mid 0}^{[1]}, \quad\left[\hat{X}_{1}^{[0]},\left(P_{0 \mid 0}^{[1]}-P_{0 \mid 0}^{[2]}\right)\right]_{1}=0 .
$$

Further:

$$
\left[\hat{T}_{2}^{[0]}, P_{1 \mid 0}^{[1]}\right]_{L}=0, \quad L=2,3 \quad \text { and } \quad\left[\hat{S}_{2}^{[0]}, P_{1 \mid 0}^{[1]}\right]_{3}=0 .
$$

Faithful to the correspondence principle as our guide, we postulate that

i) there does not exist any quantum observable which - as far as its mass dimensions are concerned - scales like a negative power of $\left(\frac{\hbar}{2 \pi \alpha^{\prime}}\right)$;

ii) $\frac{1}{\left(\hbar / 2 \pi \alpha^{\prime}\right)}$ times the commutator of two quantum observables produces a quantum observable or zero;

iii) there exists a one to one correspondence between the relations among the classical observables contained in the classical $*$-algebras $\mathfrak{h}^{[k]-}, k=0,1,2$, and the relations among the quantum observables contained in the quantum $*$-algebras $\hat{\mathfrak{h}}^{[k]-}, k=$ $0,1,2$. 
Up to quantum corrections, the quantum relations can be obtained from the classical relations according to the following rules:

$\alpha$ ) replace each (simple) Poisson bracket $\{\cdot, \cdot\}_{j}$ of the classical irreducible tensor variables by $\left(\frac{i \hbar}{2 \pi \alpha^{\prime}}\right)^{-1}$ times the commutator $[\cdot, \cdot]_{j}$ of their respective (original) counterparts in $\hat{\mathfrak{h}}^{[k]-}, k=0,1,2$, without changing the original succession of brackets inside an iterated bracket;

$\beta$ ) replace each (simple) product $(\cdot, \cdot)_{j}$ of the classical irreducible tensor variables by $\frac{1}{2}$ times the anticommutator $[\cdot, \cdot]_{j}^{+}$of their respective (original) counterparts in $\hat{\mathfrak{h}}^{[k]-}, k=0,1,2$, without changing the original succession of brackets inside an iterated bracket.

Now that the Poisson bracket action has been replaced by the commutator action $[\cdot, \cdot]_{j}$, the symbol $\{\cdot, \cdot\}_{j}$ is available anew. From now on, we shall employ the curly brackets $\{\cdot, \cdot\}_{j}$ (rather than the straight brackets $[\cdot, \cdot]_{j}^{+}$) as the symbol for the anticommutator.

The quantum corrections are distinguished from the so-called classical parts of the quantum relations (just constructed) in that the coefficients of the irreducible tensor operators appearing in these corrections display explicit positive integer powers in $\left(\frac{\hbar}{2 \pi \alpha^{\prime}}\right)$.

The above replacement of products by anticommutators has significant advantages over alternative replacements because of the relative simplicity of the recoupling formulae for commutators, anticommutators and a mix of both involving three irreducible tensor operators, $c f$. Ref. [1].

Moreover, manipulations of operator-valued dynamical variables in terms of commutators and anticommutators differ from those of the corresponding classical dynamical variables in terms of Poisson brackets and anticommutators by double commutators only (cf. Ref. [1]).

The quantization of the composition law $\left(\mathcal{A}_{1 \mid 0} \cdot P_{1 \mid 0}^{[1]}\right)_{0}=0$ does not pose any problems. In quantum theory it assumes the shape

$$
\left\{\hat{\mathcal{A}}_{1 \mid 0}, P_{1 \mid 0}^{[1]}\right\}_{0}=0
$$

with

$$
\begin{aligned}
& \hat{\mathcal{A}}_{1 \mid 0}:=-i \hat{J}_{1}^{[0]}\left(P_{0 \mid 0}^{[1]} \cdot P_{0 \mid 0}^{[2]}\right)+\hat{W}_{1 \mid 0}^{[1]} P_{0 \mid 0}^{[2]}+\hat{W}_{1 \mid 0}^{[2]} P_{0 \mid 0}^{[1]} \\
& \hat{\mathcal{B}}_{1 \mid 0}:=-i \hat{J}_{1}^{[0]}\left(P_{0 \mid 0}^{[1]}-P_{0 \mid 0}^{[2]}\right)+\hat{W}_{1 \mid 0}^{[1]}-\hat{W}_{1 \mid 0}^{[2]}
\end{aligned}
$$

where

$$
\hat{W}_{1 \mid 0}^{[k]}:=i\left[\hat{J}_{1}^{[k]} \mathfrak{m}^{[k]}+\frac{1}{2}\left\{\hat{J}_{1}^{[k]}, P_{1 \mid 0}^{[\neq k]}\right\}_{0} \delta^{[k]} P_{1 \mid 0}^{[k]}\right] .
$$

In order to explore the chances for a consistent quantization of the composition laws (11) and (2) and to narrow down their likely appearances as operator relations, we resort to the scrutiny of all conceivable preconditions they have to satisfy in quantum theory.

Apart from the requirements related to covariance, hermiticity, exchange (anti)symmetry and to the correspondence principle, these preconditions include the observance of

a) (an adequate quantal version of) the kinematical constraints, 
b) (an adequate quantal version of) the dynamical constraints.

The requirements related to covariance (both with respect to the Lorentz and to the $\mathfrak{V}^{[0]}$ algebras, respectively), hermiticity, exchange (anti)symmetry and to the correspondence principle for both sides of the composition laws are easily met (see above):

starting with the right hand sides, the r.h.s. of (1) goes over into

$$
\begin{array}{r}
{\left[\hat{\Xi}_{2 \mid 0}^{[1]}-\hat{\Xi}_{2 \mid 0}^{[2]}\right] \frac{1}{\mathfrak{m}^{[0]}}-4\left(\hat{\mathcal{A}}_{1 \mid 0}^{2}\right)_{2}\left\langle\frac{\left(P_{0 \mid 0}^{[1]}-P_{0 \mid 0}^{[2]}\right)}{\left(P_{0 \mid 0}^{[1]} \cdot P_{0 \mid 0}^{[2]}\right)^{2}}\right\rangle+4\left\{\hat{\mathcal{A}}_{1 \mid 0}, \hat{\mathcal{B}}_{1 \mid 0}\right\}_{2}\left\langle\frac{1}{\left(P_{0 \mid 0}^{[1]} \cdot P_{0 \mid 0}^{[2]}\right)}\right\rangle+} \\
h^{2}\left(P_{1 \mid 0}^{[1] 2}\right)_{2}\left\langle\frac{\left(P_{0 \mid 0}^{[1]}-P_{0 \mid 0}^{[2]}\right)}{\mathfrak{m}^{[0] 2}} \xi\left(\left(\frac{\left(P_{0 \mid 0}^{[1]}-P_{0 \mid 0}^{[2]}\right)}{\mathfrak{m}^{[0]}}\right)^{2}, \frac{\left(P_{1 \mid 0}^{[1] 2}\right)_{0}}{\mathfrak{m}^{[0] 2}}\right)\right\rangle,
\end{array}
$$

the r.h.s. of (2) goes over into

$$
\left[\hat{\Theta}_{2 \mid 0}^{[1]}+\hat{\Theta}_{2 \mid 0}^{[2]}\right] \frac{1}{\mathfrak{m}^{[0]}}+12\left(\hat{\mathcal{A}}_{1 \mid 0}^{2}\right)_{2}\left\langle\frac{1}{\left(P_{0 \mid 0}^{[1]} \cdot P_{0 \mid 0}^{[2]}\right)}\right\rangle+h^{2}\left(P_{1 \mid 0}^{[1] 2}\right)_{2}\left\langle\vartheta\left(\left(\frac{\left(P_{0 \mid 0}^{[1]}-P_{0 \mid 0}^{[2]}\right)}{\mathfrak{m}^{[0]}}\right)^{2}, \frac{\left(P_{1 \mid 0}^{[1] 2}\right)_{0}}{\mathfrak{m}^{[0] 2}}\right)\right\rangle .
$$

Here and in the sequel the symbol $\mathrm{h}$ stands for the fraction $\frac{\hbar}{2 \pi \alpha^{\prime}}$. The above irreducible tensor operators $\hat{\Xi}_{2 \mid 0}^{[k]}$ and $\hat{\Theta}_{2 \mid 0}^{[k]}, k=1,2$, are obtained from the irreducible tensor variables $\Xi_{2 \mid 0}^{[k]}$ and $\Theta_{2 \mid 0}^{[k]}$, respectively, by replacing

$X_{2}^{[k]} \quad$ by $\quad X_{2}^{[k]}=-i \sqrt{\frac{3}{2}}\left\{\hat{S}_{2}^{[k]}, P_{1 \mid k}^{[\neq k]}\right\}_{2}+\frac{1}{2}\left\{\hat{S}_{1}^{[k]}, P_{1 \mid k}^{[\neq k]}\right\}_{2}$,

$Z_{2}^{[k]} \quad$ by $\quad z_{2}^{[k]}=-\sqrt{3}\left\{\hat{T}_{2}^{[k]},\left(P_{1 \mid k}^{[\neq k] 2}\right)_{0}\right\}_{2}+\sqrt{21}\left\{\hat{T}_{2}^{[k]},\left(P_{1 \mid k}^{[\neq k] 2}\right)_{2}\right)_{2}-\frac{1}{2}\left\{\hat{B}_{0}^{(1)[k]},\left(P_{1 \mid k}^{[\neq k] 2}\right)_{2}\right\}_{2}$,

$k=1,2,\left(\mathcal{A}_{1 \mid 0}^{2}\right)_{2}$ by $\left(\hat{\mathcal{A}}_{1 \mid 0}^{2}\right)_{2}$ and $\left(\mathcal{A}_{1 \mid 0} \cdot \mathcal{B}_{1 \mid 0}\right)_{2}$ by $\frac{1}{2}\left\{\hat{\mathcal{A}}_{1 \mid 0}, \hat{\mathcal{B}}_{1 \mid 0}\right\}_{2}$.

$\xi\left(\left(\frac{\left(P_{0 \mid 0}^{[1]}-P_{0 \mid 0}^{[2]}\right)}{\mathfrak{m}^{[0]}}\right)^{2}, \frac{\left(P_{1 \mid 0}^{[1] 2}\right)_{0}}{\mathfrak{m}^{[0] 2}}\right)$ and $\vartheta\left(\left(\frac{\left(P_{0 \mid 0}^{[1]}-P_{0 \mid 0}^{[2]}\right)}{\mathfrak{m}^{[0]}}\right)^{2}, \frac{\left(P_{1 \mid 0}^{[1] 2}\right)_{0}}{\mathfrak{m}^{[0] 2}}\right)$ are scalar, real, as yet undetermined numerical functions of their dimensionless arguments, functions which are symmetric under the exchange of the branches [1] and [2].

Turning to the left hand sides of the composition laws (11) and (2), or rather to the equations defining them, the symbol $X_{2}^{[0]}$ on the l.h.s. of (11) is defined by

$$
X_{2}^{[0]}:=-i \sqrt{\frac{3}{2}}\left\{\hat{S}_{2}^{[0]}, P_{1 \mid 0}^{[1]}\right\}_{2}+\frac{1}{2}\left\{\hat{S}_{1}^{[0]}, P_{1 \mid 0}^{[1]}\right\}_{2},
$$

while the symbol $Z_{2}^{[0]}$ on the l.h.s. of (2) is replaced by

$$
z_{2}^{[0]}:=\frac{9}{4}\left\{\left\{\hat{T}_{2}^{[0]}, P_{1 \mid 0}^{[1]}\right\}_{2}, P_{1 \mid 0}^{[1]}\right\}_{2}+\frac{1}{4} \sqrt{15}\left\{\left\{\hat{T}_{2}^{[0]}, P_{1 \mid 0}^{[1]}\right\}_{1}, P_{1 \mid 0}^{[1]}\right\}_{2}-\frac{1}{4}\left\{\left\{\hat{B}_{0}^{(1)[0]}, P_{1 \mid 0}^{[1]}\right\}_{1}, P_{1 \mid 0}^{[1]}\right\}_{2} .
$$

This way of defining $z_{2}^{[0]}$ ensures that the kinematical constraint for the composition law (2) is identically satisfied for both sides of (2) in the form:

$$
\begin{gathered}
\left\{\left\{z_{2}^{[0]}, P_{1 \mid 0}^{[1]}\right\}_{1}, P_{1 \mid 0}^{[1]}\right\}_{1}=0, \\
\left\{\left\{\frac{1}{\mathfrak{m}^{[0]}}\left[\hat{\Theta}_{2 \mid 0}^{[1]}+\hat{\Theta}_{2 \mid 0}^{[2]}\right]+\frac{12}{\left(P_{0 \mid 0}^{[1]} \cdot P_{0 \mid 0}^{[2]}\right)}\left(\hat{\mathcal{A}}_{1 \mid 0}^{2}\right)_{2}+h^{2}\left(P_{1 \mid 0}^{[1] 2}\right)_{2}\left\langle\vartheta\left(\left(\frac{\left(P_{0 \mid 0}^{[1]}-P_{0 \mid 0}^{[2]}\right)}{\mathfrak{m}^{[0]}}\right)^{2}, \frac{\left(P_{1] 0}^{[1] 2}\right)_{0}}{\mathfrak{m}^{[0] 2}}\right)\right\rangle,\right.\right. \\
\left.\left.P_{1 \mid 0}^{[1]}\right\}_{1}, P_{1 \mid 0}^{[1]}\right\}_{1}=0 .
\end{gathered}
$$


(This is also true, if the intermediate spin-channel index 1 is replaced by the intermediate spin-channel index 2.)

As before, we define the commutators $\left[\mathcal{X}_{2}^{[0]}, P_{1 \mid 0}^{[1]}\right]_{L},\left[\mathcal{Z}_{2}^{[0]}, P_{1 \mid 0}^{[1]}\right]_{L}, L=1,2,3,\left[X_{2}^{[0]},\left(P_{0 \mid 0}^{[1]}-\right.\right.$ $\left.\left.P_{0 \mid 0}^{[2]}\right)\right]_{2}$ and $\left[Z_{2}^{[0]},\left(P_{0 \mid 0}^{[1]}-P_{0 \mid 0}^{[2]}\right)\right]_{2}$ in accordance with the right hand sides of their composition laws. In this way, we are led to specifications of the commutators just mentioned which literally agree with the application of substitution rules $\alpha$ ) and $\beta$ ) to the specifications of the corresponding Poisson brackets.

Next, we replace the subset of generators of $\hat{\mathfrak{h}}^{[0]-}: \hat{B}_{0}^{(1)[0]}, \hat{S}_{1}^{[0]}, \hat{S}_{2}^{[0]}, \hat{T}_{2}^{[0]}$ by the following equivalent subset of irreducible tensor operators:

$$
\hat{s}_{1}:=\left\{\hat{S}_{2}^{[0]}, P_{1 \mid 0}^{[1]}\right\}_{1} \quad, \quad X_{2}^{[0]} ; \quad \hat{t}_{1}:=\left\{\hat{T}_{2}^{[0]}, P_{1 \mid 0}^{[1]}\right\}_{1} \quad, \quad z_{2}^{[0]} .
$$

Further, using the formal properties of the commutators, we convert our various specifications of the "mixed" commutators into the equivalent form

$$
\begin{aligned}
& \mathcal{C}_{2}:=\left[\hat{s}_{1}, P_{1 \mid 0}^{[1]}\right]_{2}=\frac{1}{14}\left\{\mathfrak{C}_{1}, \frac{\left(P_{10}^{[1] 2}\right)_{2}}{\left(P_{1 \mid 0}^{[1] 2}\right)_{0}}\right\}_{2}+\frac{1}{20}\left\{\mathfrak{C}_{0}, \frac{\left(P_{1 \mid 0}^{[1] 2}\right)_{2}}{\left(P_{1 \mid 0}^{1 \mid 12}\right)_{0}}\right\}_{2}- \\
& \frac{24}{35} \sqrt{\frac{3}{5}} \text { h }\left\{\hat{\mathcal{B}}_{1 \mid 0}, P_{1 \mid 0}^{[1]}\right\}_{2}+\frac{8}{5 \sqrt{7}} \text { h }\left\{\hat{\mathcal{B}}_{1 \mid 0}, \frac{\left(P_{1 \mid 0}^{[1] 3}\right)_{3}}{\left(P_{1 \mid 0}^{[1] 2}\right)_{0}}\right\}_{2}, \\
& \mathcal{C}_{1}:=\left[\hat{s}_{1}, P_{1 \mid 0}^{[1]}\right]_{1}, \\
& \mathcal{C}_{0}:=\left[\hat{s}_{1}, P_{1 \mid 0}^{[1]}\right]_{0}, \\
& {\left[X_{2}^{[0]}, P_{1 \mid 0}^{[1]}\right]_{L}= \begin{cases}i 2 \sqrt{\frac{10}{3}} \mathrm{~h}\left\{\hat{\mathcal{B}}_{1 \mid 0}, P_{1 \mid 0}^{[1]}\right\}_{1} & \text { for } L=1 \\
i 2 \sqrt{6} \mathrm{~h}\left\{\hat{\mathcal{B}}_{1 \mid 0}, P_{1 \mid 0}^{[1]}\right\}_{2} & \text { for } L=2 \\
0 & \text { for } L=3\end{cases} } \\
& \mathcal{K}_{2}:=\left[\hat{t}_{1}, P_{1 \mid 0}^{[1]}\right]_{2}=-i \sqrt{\frac{2}{15}} \mathrm{~h}\left\{\hat{\mathcal{A}}_{1 \mid 0}, \frac{\left(P_{10}^{[1] 2}\right)_{2}}{\left(P_{1 \mid 0}^{[1] 2}\right)_{0}}\right\}_{2}+\frac{1}{20}\left\{\mathcal{K}_{0}, \frac{\left(P_{1 \mid 0}^{[1] 2}\right)_{2}}{\left(P_{1 \mid 0}^{1 \mid 2}\right)_{0}}\right\}_{2}, \\
& \mathcal{K}_{1}:=\left[\hat{t}_{1}, P_{1 \mid 0]}^{[1]}\right]_{1}=i 2 \sqrt{\frac{10}{3}} \text { h } \hat{\mathcal{A}}_{1 \mid 0}, \\
& \mathcal{K}_{0}:=\left[\hat{t}_{1}, P_{1 \mid 0}^{[1]}\right]_{0} \text {, } \\
& {\left[z_{2}^{[0]}, P_{1 \mid 0}^{[1]}\right]_{L}= \begin{cases}i 2 \sqrt{30} \mathrm{~h}\left\{\hat{\mathcal{A}}_{1 \mid 0}, P_{1 \mid 0}^{[1]}\right\}_{1} & \text { for } L=1 \\
i 6 \sqrt{6} \mathrm{~h}\left\{\hat{\mathcal{A}}_{1 \mid 0}, P_{1 \mid 0}^{[1]}\right\}_{2} & \text { for } L=2 \\
0 & \text { for } L=3\end{cases} }
\end{aligned}
$$

With the help of the above definitions of $\mathcal{C}_{1}, \mathcal{C}_{0}$ and $\mathcal{K}_{0}$, we can express the original 
generators in terms of $\hat{s}_{1}, x_{2}^{[0]} ; \hat{t}_{1}, z_{2}^{[0]}$ :

$$
\begin{aligned}
& \hat{S}_{1}^{[0]}=i \frac{1}{3} \sqrt{\frac{5}{2}}\left\{\hat{s}_{1}, \frac{P_{1 \mid 0}^{[1]}}{\left(P_{1 \mid 0}^{[1] 2}\right)_{0}}\right\}_{1}+\frac{3}{2 \sqrt{5}}\left\{X_{2}^{[0]}, \frac{P_{1 \mid 0}^{[1]}}{\left(P_{1 \mid 0}^{[1] 2}\right)_{0}}\right\}_{1}-\frac{1}{12} \sqrt{\frac{7}{3}}\left\{X_{2}^{[0]}, \frac{\left(P_{1 \mid 0}^{[1] 3}\right)_{3}}{\left(P_{1 \mid 0}^{[1] 2}\right)_{0}{ }^{2}}\right\}_{1}+ \\
& i \frac{43}{630 \sqrt{2}}\left\{\left[\mathcal{C}_{1}, P_{1 \mid 0}^{[1]}\right]_{2}, \frac{P_{1 \mid 0}^{[1]}}{\left(P_{1 \mid 0}^{[1] 2}\right)_{0}{ }^{2}}\right\}_{1}+i \frac{11}{9} \sqrt{\frac{2}{105}}\left\{\left[\mathcal{C}_{1}, P_{1 \mid 0}^{[1]}\right]_{2}, \frac{\left(P_{1 \mid 0}^{[1] 3}\right)_{3}}{\left(P_{1 \mid 0}^{[1] 2}\right)_{0}{ }^{3}}\right\}_{1}- \\
& i \frac{5}{63} \sqrt{\frac{5}{6}}\left\{\left[\mathcal{C}_{1}, P_{1 \mid 0}^{[1]}\right]_{1}, \frac{P_{1 \mid 0}^{[1]}}{\left(P_{1 \mid 0}^{[1] 2}\right)_{0}^{2}}\right\}_{1}+i \frac{7}{180 \sqrt{10}}\left\{\left[\mathcal{C}_{0}, P_{1 \mid 0}^{[1]}\right]_{1}, \frac{P_{1 \mid 0}^{[1]}}{\left(P_{1 \mid 0}^{[1] 2}\right)_{0}^{2}}\right\}_{1}+ \\
& \sqrt{3} h^{2} P_{1 \mid 0}^{[1]} \frac{\left(P_{0 \mid 0}^{[1]}-P_{0 \mid 0}^{[2]}\right)}{\left(P_{1 \mid 0}^{[1] 2}\right)_{0}} \\
& \hat{S}_{2}^{[0]}=\frac{3}{4 \sqrt{5}}\left\{\hat{s}_{1}, \frac{P_{1 \mid 0}^{[1]}}{\left(P_{1 \mid 0}^{[1] 2}\right)_{0}}\right\}_{2}-\frac{1}{24} \sqrt{\frac{7}{3}}\left\{\hat{s}_{1}, \frac{\left(P_{1 \mid 0}^{[1] 3}\right)_{3}}{\left(P_{1 \mid 0}^{[1] 2}\right)_{0}^{2}}\right\}_{2}-i \frac{1}{5 \sqrt{2}}\left\{X_{2}^{[0]}, \frac{P_{1 \mid 0}^{[1]}}{\left(P_{1 \mid 0}^{[1] 2}\right)_{0}}\right\}_{2}+ \\
& i \frac{1}{12} \sqrt{\frac{7}{5}}\left\{X_{2}^{[0]}, \frac{\left(P_{1 \mid 0}^{[1] 3}\right)_{3}}{\left(P_{1 \mid 0}^{[1] 2}\right)_{0}^{2}}\right\}_{2}-\frac{3}{560 \sqrt{5}}\left\{\left[\mathcal{C}_{1}, P_{1 \mid 0}^{[1]}\right]_{2}, \frac{P_{1 \mid 0}^{[1]}}{\left(P_{1 \mid 0}^{[1] 2}\right)_{0}{ }^{2}}\right\}_{2}+ \\
& \frac{257}{720 \sqrt{14}}\left\{\left[\mathcal{C}_{1}, P_{1 \mid 0}^{[1]}\right]_{2}, \frac{\left(P_{1 \mid 0}^{[1] 3}\right)_{3}}{\left(P_{1 \mid 0}^{[1] 2}\right)_{0}{ }^{3}}\right\}_{2}-\frac{11}{280} \sqrt{\frac{3}{5}}\left\{\left[\mathcal{C}_{1}, P_{1 \mid 0}^{[1]}\right]_{1}, \frac{P_{100}^{[1]}}{\left(P_{1 \mid 0}^{[1] 2}\right)_{0}{ }^{2}}\right\}_{2}- \\
& \frac{73}{1440 \sqrt{7}}\left\{\left[\mathcal{C}_{1}, P_{1 \mid 0}^{[1]}\right]_{1}, \frac{\left(P_{1 \mid 0}^{[1] 3}\right)_{3}}{\left(P_{1 \mid 0}^{[1] 2}\right)_{0}{ }^{3}}\right\}_{2}+\frac{57}{800 \sqrt{5}}\left\{\left[\mathcal{C}_{0}, P_{1 \mid 0}^{[1]}\right]_{1}, \frac{P_{1 \mid 0}^{[1]}}{\left(P_{1 \mid 0}^{[1] 2}\right)_{0}{ }^{2}}\right\}_{2}- \\
& \frac{409}{4800} \sqrt{\frac{7}{3}}\left\{\left[\mathcal{C}_{0}, P_{1 \mid 0}^{[1]}\right]_{1}, \frac{\left(P_{1 \mid 0}^{[1] 3}\right)_{3}}{\left(P_{1 \mid 0}^{[1] 2}\right)_{0}^{3}}\right\}_{2}, \\
& \hat{B}_{0}^{(1)[0]}=\frac{\sqrt{15}}{4}\left\{\hat{t}_{1}, \frac{P_{1 \mid 0}^{[1]}}{\left(P_{1 \mid 0}^{[1] 2}\right)_{0}}\right\}_{0}-\frac{\sqrt{5}}{4}\left\{z_{2}^{[0]}, \frac{\left(P_{1 \mid 0}^{[1] 2}\right)_{2}}{\left(P_{1 \mid 0}^{[1] 2}\right)_{0}^{2}}\right\}_{0}+ \\
& \frac{11}{80} \sqrt{\frac{3}{5}}\left\{\left[\mathcal{K}_{0}, P_{1 \mid 0}^{[1]}\right]_{1}, \frac{P_{110}^{[1]}}{\left(P_{1 \mid 0}^{[1] 2}\right)_{0}{ }^{2}}\right\}_{0}+\frac{16}{5 \sqrt{3}} h^{2}\left(P_{1 \mid 0}^{[1] 2}\right)_{0} \frac{\left(P_{0 \mid 0}^{[1]} \cdot P_{0 \mid 0}^{[2]}\right)}{\left(P_{1 \mid 0}^{[1] 2}\right)_{0}{ }^{2}} \\
& \hat{T}_{2}^{[0]}=\frac{3}{4 \sqrt{5}}\left\{\hat{t}_{1}, \frac{P_{1 \mid 0}^{[1]}}{\left(P_{1 \mid 0}^{[1] 2}\right)_{0}}\right\}_{2}-\frac{1}{24} \sqrt{\frac{7}{3}}\left\{\hat{t}_{1}, \frac{\left(P_{1 \mid 0}^{[1] 3}\right)_{3}}{\left(P_{1 \mid 0}^{[1] 2}\right)_{0}{ }^{2}}\right\}_{2}-\frac{1}{24 \sqrt{3}}\left\{z_{2}^{[0]}, \frac{1}{\left(P_{1 \mid 0}^{[1] 2}\right)_{0}}\right\}_{2}+ \\
& \frac{1}{48} \sqrt{\frac{7}{3}}\left\{Z_{2}^{[0]}, \frac{\left(P_{10 \mid}^{[1] 2}\right)_{2}}{\left(P_{1 \mid 0}^{[1] 2}\right)_{0}^{2}}\right\}_{2}+\frac{57}{800 \sqrt{5}}\left\{\left[\mathcal{K}_{0}, P_{1 \mid 0}^{[1]}\right] 1, \frac{P_{100}^{[1]}}{\left(P_{1 \mid 0}^{[1] 2}\right)_{0}^{2}}\right\}_{2}- \\
& \frac{409}{4800} \sqrt{\frac{7}{3}}\left\{\left[\mathcal{K}_{0}, P_{1 \mid 0}^{[1]}\right]_{1}, \frac{\left(P_{1 \mid 0}^{[1] 3}\right)_{3}}{\left(P_{1 \mid 0}^{[1] 2}\right)_{0}{ }^{3}}\right\}_{2}+\frac{19}{15} \mathrm{~h}^{2}\left(P_{1 \mid 0}^{[1] 2}\right)_{2} \frac{\left(P_{0 \mid 0}^{[1]} \cdot P_{0 \mid 0}^{[2]}\right)}{\left(P_{1 \mid 0}^{[1] 2}\right)_{0}^{2}} .
\end{aligned}
$$

Formally, the errors committed in the course of the corresponding manipulations involve operators scaling like $\mathrm{h}^{-2}$. Such operators do not represent observables. Hence, the errors are just spurious.

We are now ready to tackle the last and decisive issue, the clarification of the question whether or not the quantal composition laws $X_{2}^{[0]}=\ldots$ and $z_{2}^{[0]}=\ldots$ are in agreement 
with the generalized commutation relations

$$
\begin{aligned}
{\left[\hat{T}_{2}^{[k]}, \hat{T}_{2}^{[k]}\right]_{3}=} & -i\left[\hat{S}_{2}^{[k]}, \hat{S}_{1}^{[k]}\right]_{3}-8 \mathrm{~h}\left\{\hat{J}_{1}^{[k]},\left(\hat{J}_{1}^{[k]}\right)_{2}\right\}_{3}, \\
{\left[\hat{S}_{2}^{[k]}, \hat{S}_{2}^{[k]}\right]_{3}=} & i 2\left[\hat{S}_{2}^{[k]}, \hat{S}_{1}^{[k]}\right]_{3}+4 \mathrm{~h}\left\{\hat{J}_{1}^{[k]}, \hat{T}_{2}^{[k]}\right\}_{3}+24 \mathrm{~h}\left\{\hat{J}_{1}^{[k]},\left(\hat{J}_{1}^{[k]}\right)_{2}\right\}_{3}, \\
{\left[\hat{S}_{2}^{[k]}, \hat{S}_{2}^{[k]}\right]_{1}=} & -i \sqrt{\frac{2}{3}}\left[\hat{S}_{2}^{[k]}, \hat{S}_{1}^{[k]}\right]_{1}-\frac{1}{6} \sqrt{5}\left[\hat{S}_{1}^{[k]}, \hat{S}_{1}^{[k]}\right]_{1}+ \\
& 8 \sqrt{\frac{2}{3}} \mathrm{~h}\left\{\hat{J}_{1}^{[k]}, \hat{T}_{2}^{[k]}\right\}_{1}+16 \sqrt{\frac{2}{15}} \mathrm{~h}\left\{\hat{J}_{1}^{[k]},\left(\hat{J}_{1}^{[k]}\right)_{0}\right\}_{1}-\mathrm{h}^{3} f \sqrt{10} \hat{J}_{1}^{[k]}, \\
{\left[\hat{B}_{0}^{(1)[k]}, \hat{T}_{2}^{[k]}\right]_{2}=} & i \sqrt{6}\left[\hat{S}_{2}^{[k]}, \hat{S}_{1}^{[k]}\right]_{2}, \\
{\left[\hat{T}_{2}^{[k]}, \hat{S}_{2}^{[k]}\right]_{4}=} & 0, \\
{\left[\hat{T}_{2}^{[k]}, \hat{S}_{2}^{[k]}\right]_{3}=} & i\left[\hat{T}_{2}^{[k]}, \hat{S}_{1}^{[k]}\right]_{3}-4 \mathrm{~h}\left\{\hat{J}_{1}^{[k]}, \hat{S}_{2}^{[k]}\right\}_{3}, \\
{\left[\hat{T}_{2}^{[k]}, \hat{S}_{2}^{[k]}\right]_{2}=} & -i \frac{1}{3} \sqrt{\frac{7}{2}}\left[\hat{T}_{2}^{[k]}, \hat{S}_{1}^{[k]}\right]_{2}+\frac{2}{3} \sqrt{14} \mathrm{~h}\left\{\hat{J}_{1}^{[k]}, \hat{S}_{2}^{[k]}\right\}_{2}, \\
{\left[\hat{B}_{0}^{(1)[k]}, \hat{S}_{2}^{[k]}\right]_{2}=} & -i 2 \sqrt{\frac{2}{3}}\left[\hat{T}_{2}^{[k]}, \hat{S}_{1}^{[k]}\right]_{2}+2 \sqrt{\frac{2}{3}} \mathrm{~h}\left\{\hat{J}_{1}^{[k]}, \hat{S}_{2}^{[k]}\right\}_{2}+i 6 \mathrm{~h}\left\{\hat{J}_{1}^{[k]}, \hat{S}_{1}^{[k]}\right\}_{2}, \\
{\left[\hat{B}_{0}^{(1)[k]}, \hat{S}_{1}^{[k]}\right]_{1}=} & -i 6 \sqrt{\frac{2}{5}}\left[\hat{T}_{2}^{[k]}, \hat{S}_{2}^{[k]}\right]_{1}+2 \sqrt{\frac{3}{5}}\left[\hat{T}_{2}^{[k]}, \hat{S}_{1}^{[k]}\right]_{1}- \\
& i 12 \sqrt{\frac{3}{5}} \mathrm{~h}\left\{\hat{J}_{1}^{[k]}, \hat{S}_{2}^{[k]}\right\}_{1}-6 \sqrt{2} \mathrm{~h}\left\{\hat{J}_{1}^{[k]}, \hat{S}_{1}^{[k]}\right\}_{1},
\end{aligned}
$$

$f$ being a free rational common parameter, $k=0,1,2$. In other words, do the two alternative procedures described below give concordant results with (or without) the help of suitable choices of the functions $\xi$ and $\vartheta$ ?

The two alternative procedures consist of a separate and a common part: Replace in the expressions DYN0, ., DYN3 in turns the tensor variables $X_{2}^{[0]}$ and $Z_{2}^{[0]}$

a) by their respective quantal counterparts $X_{2}^{[0]}$ and $z_{2}^{[0]}$

b) by the right hand side of the respective quantal composition laws $X_{2}^{[0]}=\ldots$ and $z_{2}^{[0]}=\ldots$

Subsequently apply the substitution rules $\alpha$ ) and $\beta$ ) and evaluate the commutators with the help of the generalized commutation relations. Finally, use the recoupling formulae of Ref. [1] for a suitable presentation of the respective results.

The procedures $a$ ) and $b$ ) involve among other things the use of the recoupling formula which regulates the replacement of the double anticommutator $\left\{\left\{A_{j_{1}}, B_{j_{2}}\right\}_{l}, C_{j_{3}}\right\}_{L}$ by a linear combination of double anticommutators with cyclically permuted entries plus a linear combination of double commutators. That part of each individual result of procedure $a$ ) and of procedure $b$ ) which is due to the occurrence of such double commutators is called the "subleading part" of the result of the respective procedure. Its complement is called the "leading part" of the result of the respective procedure.

An explicit factor $\mathrm{h}^{2}$ could be extracted from each subleading part in keeping with the regular scaling behaviour of the remainder when $h$ tends to zero. The replacement of commutators $[\cdot, \cdot]_{j}$ by "adapted" commutators $\frac{1}{h}[\cdot, \cdot]_{j}$ is all that would be required.

While there does not exist any difficulty concerning the execution of procedure $b$ ) (it turns out that the results are unaffected by special choices of the functions $\xi$ and $\vartheta$ ), the execution of procedure $a$ ) requires some reflection. For carrying out the latter procedure, 
especially when evaluating the commutators $\left[X_{2}^{[0]}, X_{2}^{[0]}\right]_{L},\left[Z_{2}^{[0]}, Z_{2}^{[0]}\right]_{L}$ and $\left[Z_{2}^{[0]}, X_{2}^{[0]}\right]_{L}$, two strategies come to mind.

Strategy no 1 (explained for the cases DYN1, .., DYN3)

In the leading part, commutator by commutator, at the expense of contributions from terms $\left\{\left[X_{2}^{[0]},\left(P_{1 \mid 0}^{[1] 2}\right)_{2}\right]_{L}, \hat{B}_{0}^{(1)[0]}\right\}_{L}, \quad\left\{\left[X_{2}^{[0]},\left(P_{1 \mid 0}^{[1] 2}\right)_{2}\right]_{k}, \hat{T}_{2}^{[0]}\right\}_{L} \quad$ and $\quad\left\{\left[X_{2}^{[0]},\left(P_{1 \mid 0}^{[1] 2}\right)_{0}\right]_{2}, \hat{T}_{2}^{[0]}\right\}_{L}$ $\left(\left\{\left[Z_{2}^{[0]}, P_{1 \mid 0}^{[1]}\right]_{k}, \hat{S}_{1}^{[0]}\right\}_{L}\right.$ and $\left.\left\{\left[Z_{2}^{[0]}, P_{1 \mid 0}^{[1]}\right]_{k}, \hat{S}_{2}^{[0]}\right\}_{L}\right)$, move the irreducible tensor operator $X_{2}^{[0]}$ $\left(Z_{2}^{[0]}\right)$ as a whole into commutator brackets pairing it with the generators $\hat{B}_{0}^{(1)[0]}$ and $\hat{T}_{2}^{[0]}\left(\hat{S}_{1}^{[0]}\right.$ and $\left.\hat{S}_{2}^{[0]}\right)$, originally contained inside the irreducible tensor operator $z_{2}^{[0]}\left(X_{2}^{[0]}\right)$. Left with a linear combination of terms $\left\{\left[X_{2}^{[0]}, \hat{B}_{0}^{(1)[0]}\right]_{2},\left(P_{1 \mid 0}^{[1] 2}\right)_{2}\right\}_{L},\left\{\left[X_{2}^{[0]}, \hat{T}_{2}^{[0]}\right]_{k},\left(P_{1 \mid 0}^{[1] 2}\right)_{2}\right\}_{L}$ and $\left\{\left[\mathcal{X}_{2}^{[0]}, \hat{T}_{2}^{[0]}\right]_{L},\left(P_{1 \mid 0}^{[1] 2}\right)_{0}\right\}_{L}\left(\left\{\left[z_{2}^{[0]}, \hat{S}_{1}^{[0]}\right]_{k}, P_{1 \mid 0}^{[1]}\right\}_{L}\right.$ and $\left.\left\{\left[Z_{2}^{[0]}, \hat{S}_{2}^{[0]}\right]_{k}, P_{1 \mid 0}^{[1]}\right\}_{L}\right)$ apply the Leibniz rule, i.e. the recoupling formula for a commutator containing an anticommutator. The outcome is a sum of two types of linear combinations: The type-one linear combination consists of terms in which $\hat{B}_{0}^{(1)[0]}$ and $\hat{T}_{2}^{[0]}\left(\hat{S}_{1}^{[0]}\right.$ and $\left.\hat{S}_{2}^{[0]}\right)$ are paired with $P_{1 \mid 0}^{[1]}$ $\left(\left(P_{1 \mid 0}^{[1] 2}\right)_{2}\right.$ or $\left.\left(P_{1 \mid 0}^{[1] 2}\right)_{0}\right)$ inside commutators, these commutators being paired with $\hat{S}_{1}^{[0]}$ or $\hat{S}_{2}^{[0]}\left(\hat{B}_{0}^{(1)[0]}\right.$ or $\left.\hat{T}_{2}^{[0]}\right)$ inside anticommutators.

The type-two linear combination consists of terms in which $\hat{B}_{0}^{(1)[0]}$ and $\hat{T}_{2}^{[0]}\left(\hat{S}_{1}^{[0]}\right.$ and $\left.\hat{S}_{2}^{[0]}\right)$ are paired with $\hat{S}_{1}^{[0]}$ or $\hat{S}_{2}^{[0]}\left(\hat{B}_{0}^{(1)[0]}\right.$ or $\left.\hat{T}_{2}^{[0]}\right)$ inside commutators, these commutators being paired with $P_{1 \mid 0}^{[1]}\left(\left(P_{1 \mid 0}^{[1] 2}\right)_{2}\right.$ or $\left.\left(P_{1 \mid 0}^{[1] 2}\right)_{0}\right)$ inside anticommutators.

With the help of Biedenharn's and Elliot's sum rule for $6-j$ symbols (cf. e.g. Ref. [6]) show that in the leading part the type-one linear combination can be arranged in a linear combination of terms $\left\{\left[z_{2}^{[0]}, P_{1 \mid 0}^{[1]}\right]_{k}, \hat{S}_{1}^{[0]}\right\}_{L}$ and $\left\{\left[z_{2}^{[0]}, P_{1 \mid 0}^{[1]}\right]_{k}, \hat{S}_{2}^{[0]}\right\}_{L}$, $\left(\left\{\left[X_{2}^{[0]},\left(P_{1 \mid 0}^{[1] 2}\right)_{2}\right]_{L}, \hat{B}_{0}^{(1)[0]}\right\}_{L},\left\{\left[X_{2}^{[0]},\left(P_{1 \mid 0}^{[1] 2}\right)_{2}\right]_{k}, \hat{T}_{2}^{[0]}\right\}_{L}\right.$ and $\left.\left\{\left[X_{2}^{[0]},\left(P_{1 \mid 0}^{[1] 2}\right)_{0}\right]_{2}, \hat{T}_{2}^{[0]}\right\}_{L}\right)$. Making use of $i)$ the generalized commutation relations and ii) the particular combinations of commutators $\left[Z_{2}^{[0]}, X_{2}^{[0]}\right]_{L}$ for DYN1, . . DYN3, show for each separate DYN that in its leading part the net contribution from the type-two combinations can be written as a sum of anticommutators none of which containing more than one generator from the collection of generators $\hat{B}_{0}^{(1)[0]}, \hat{T}_{2}^{[0]}, \hat{S}_{1}^{[0]}, \hat{S}_{2}^{[0]}$.

Note: The execution of procedure a) for the leading part does not require any specification of the commutators $\left[\hat{B}_{0}^{(1)[0]}, P_{1 \mid 0}^{[1]}\right]_{1}, \ldots,\left[\hat{S}_{2}^{[0]}, P_{1 \mid 0}^{[1]}\right]_{L}$ other than that of the commutators $\left[Z_{2}^{[0]}, P_{1 \mid 0}^{[1]}\right]_{L}$ and $\left[X_{2}^{[0]}, P_{1 \mid 0}^{[1]}\right]_{L}$.

Strategy no 2 Rewrite the generalized commutation relations for branch [0] in terms of $\hat{s}_{1}, X_{2}^{[0]} ; \hat{t}_{1}, z_{2}^{[0]}$ and insert the resulting version of the commutation relations into DYN0, ..., DYN3. Check that in the leading part of the DYNs after this insertion no commutators are left which cannot be cast into the form $\left[X_{2}^{[0]}, P_{1 \mid 0}^{[1]}\right]_{L}$ and $\left[Z_{2}^{[0]}, P_{1 \mid 0}^{[1]}\right]_{L}$. Moreover, after evaluation of these commutators the leading parts can be written as sums of anticommutators none of which containing the elements $\hat{s}_{1}$ and $\hat{t}_{1}$ or more than one element from the collection $X_{2}^{[0]}, Z_{2}^{[0]}$.

We observe that in accordance with the correspondence principle for each individual DYN the leading part of the result of the two procedures $a$ ) (independent of the strategy employed) and b) vanishes in both cases. All - if anything - that survives is the subleading part of the respective result. Pars pro toto we quote the surviving $\equiv$ 
subleading parts of the result of procedure $a$ ) and of procedure $b$ ) applied to DYN0, these parts being denoted by dyn0a and dyn0b, respectively. (As a second example, we quote the analogous parts dyn1a and dyn1b related to DYN1 in the appendix. While the expressions for the analogous parts dyn2a and dyn3a related to DYN2 and DYN3, respectively, are too lengthy to be quoted in this article at all, the expressions for the corresponding parts dyn $2 \mathrm{~b}$ and dyn $3 \mathrm{~b}$ are sufficently simple. They too are reproduced in the appendix.)

In order to arrive at the expressions for dyn·a (quoted and unquoted) we had to make extensive use of the formal properties of commutator brackets, of the vanishing of the commutator $\left[P_{1 \mid 0}^{[1]}, P_{1 \mid 0}^{[1]}\right]_{1}$, and of special derivates of the generalized commutation relations for branch [0]. These derivates are obtained as follows: We insert the expressions of the original generators in terms of $\hat{s}_{1}, x_{2}^{[0]} ; \hat{t}_{1}, z_{2}^{[0]}$ and commute separately the two sides of each generalized commutation relation twice with the components of the irreducible tensor variable $P_{1 \mid 0}^{[1]}$. Subsequently, we equate the corresponding results:

$$
\left[\left[(\text { l.h.s. }), P_{1 \mid 0}^{[1]}\right]_{l}, P_{1 \mid 0}^{[1]}\right]_{L}=\left[\left[(\text { r.h.s. }), P_{1 \mid 0}^{[1]}\right]_{l}, P_{1 \mid 0}^{[1]}\right]_{L}
$$

and work out the implications of these equations. As for the commutators

$$
\left[\mathcal{C}_{p}, P_{1 \mid 0}^{[1]}\right]_{L} \quad \text { and } \quad\left[\mathcal{K}_{q}, P_{1 \mid 0}^{[1]}\right]_{L}, \quad p, q=0,1,2
$$

turning up in the course of the act, we prefer to postpone the injection of the previous specifications for $\mathcal{C}_{2}, \mathcal{K}_{1}$ and $\mathcal{K}_{2}$, (and their full implications) to a later stage of the analysis. Instead, for the time being, we work under the hypothesis that, when evaluating the above commutators, appropriate powers of the tensor variable $P_{1 \mid 0}^{[1]}$ completely take care of the non-trivial $O(3)$ transformation properties of the respective results, these powers being accompanied by appropriate real functions $\bar{\sigma}, \tau_{01}, \tau_{11}$ and $\tau_{23}$ of the $O(3)-$ scalar observables $\mathfrak{m}^{[0]},\left(P_{0 \mid 0}^{[1]}-P_{0 \mid 0}^{[2]}\right)^{2}$ and $\left(P_{1 \mid 0}^{[1] 2}\right)_{0}$. The functions $\bar{\sigma}$ and $\tau_{23}$, having mass dimension 0 , can be thought of as functions of the quotients $\left(\frac{P_{0 \mid 0}^{[1]}-P_{0 \mid 0}^{[2]}}{\mathfrak{m}^{[0]}}\right)^{2}$ and $\frac{\left(P_{1 \mid 0}^{[1] 2}\right)_{0}}{\mathfrak{m}^{0 \mid 02}}$, while the functions $\tau_{01}$ and $\tau_{11}$ have mass dimension 2 .

In formulae:

$$
\begin{aligned}
& {\left[\mathcal{C}_{0}, P_{1 \mid 0}^{[1]}\right]_{1}=0} \\
& {\left[\mathcal{C}_{1}, P_{1 \mid 0}^{[1]}\right]_{L}=\sqrt{3}\left[\mathcal{C}_{2}, P_{1 \mid 0}^{[1]}\right]_{L}=\delta_{L, 2} \frac{1}{i} \mathrm{~h}^{2}\left(P_{1 \mid 0}^{[1] 2}\right)_{2}\left(P_{0 \mid 0}^{[1]}-P_{0 \mid 0}^{[2]}\right) \bar{\sigma},} \\
& {\left[\mathcal{K}_{0}, P_{1 \mid 0}^{[1]}\right]_{1}=\mathrm{h}^{2} P_{1 \mid 0}^{[1]} \tau_{01}} \\
& {\left[\mathcal{K}_{1}, P_{1 \mid 0}^{[1]}\right]_{L}=\delta_{L, 1} \mathrm{~h}^{2} P_{1 \mid 0}^{[1]} \tau_{11}} \\
& {\left[\mathcal{K}_{2}, P_{1 \mid 0}^{[1]}\right]_{L}=h^{2} \begin{cases}\frac{1}{\sqrt{5}} P_{1 \mid 0}^{[1]}\left[2 \tau_{01}-3\left(\frac{\tau_{11}}{\sqrt{3}}\right)\right] & \text { for } L=1 \\
0 & \text { for } L=2 \\
\left(P_{1 \mid 0}^{[1] 3}\right)_{3} \tau_{23} & \text { for } L=3 .\end{cases} }
\end{aligned}
$$

In this way we obtain the following preliminary expression for dyn0a (and for the other dyn·a) 


$$
\begin{aligned}
& \text { dyn0a }= \\
& \frac{1}{\mathrm{~h}}\left\langle\frac{25}{7 \sqrt{3}}\left\{\left[\mathcal{K}_{2}, \mathcal{K}_{2}\right]_{3},\left(P_{1 \mid 0}^{[1] 2}\right)_{2}\right\}_{2}-5 \sqrt{\frac{5}{42}}\left\{\left[\mathcal{K}_{2}, \mathcal{K}_{2}\right]_{3}, \frac{\left(P_{1 \mid 0}^{[1] 4}\right)_{4}}{\left(P_{1 \mid 0}^{[1] 2}\right)_{0}}\right\}_{2}+\right. \\
& 4 \sqrt{\frac{5}{21}}\left\{\left[\mathcal{K}_{2}, \mathcal{K}_{0}\right]_{2},\left(P_{1 \mid 0}^{[1] 2}\right)_{0}\right\}_{2}-\frac{20}{7} \sqrt{\frac{5}{3}}\left\{\left[\mathcal{K}_{2}, \mathcal{K}_{0}\right]_{2},\left(P_{1 \mid 0}^{[1] 2}\right)_{2}\right\}_{2}+ \\
& \left.\frac{5}{\sqrt{21}}\left\{\left[\mathcal{K}_{2}, \mathcal{K}_{0}\right]_{2}, \frac{\left(P_{1 \mid 0}^{[1] 4}\right)_{4}}{\left(P_{1 \mid 0}^{[1] 2}\right)_{0}}\right\}_{2}\right\rangle-\frac{1}{\mathrm{~h}}\left\langle\mathcal{K}_{2} \leftrightarrow \mathcal{C}_{2}, \mathcal{K}_{0} \leftrightarrow \mathcal{C}_{0}\right\rangle+ \\
& \frac{8}{\sqrt{35}} \mathrm{~h}\left\{\mathcal{K}_{2},\left\langle\sqrt{3} \tau_{01}+\tau_{11}-\sqrt{3} \tau_{23}\left(P_{1 \mid 0}^{[1] 2}\right)_{0}\right\rangle\left(P_{1 \mid 0}^{[1] 2}\right)_{0}\right\}_{2}- \\
& \frac{8}{7} \sqrt{5} \mathrm{~h}\left\{\mathcal{K}_{2},\left\langle\sqrt{3} \tau_{01}+\tau_{11}-\sqrt{3} \tau_{23}\left(P_{1 \mid 0}^{[1] 2}\right)_{0}\right\rangle\left(P_{1 \mid 0}^{[1] 2}\right)_{2}\right\}_{2}+ \\
& \frac{2}{\sqrt{7}} \mathrm{~h}\left\{\mathcal{K}_{2},\left\langle\sqrt{3} \tau_{01}+\tau_{11}-\sqrt{3} \tau_{23}\left(P_{1 \mid 0}^{[1] 2}\right)_{0}\right\rangle \frac{\left(P_{1 \mid 0}^{[1] 4}\right)_{4}}{\left(P_{1 \mid 0}^{[1] 2}\right)_{0}}\right\}_{2}+ \\
& i 72 \sqrt{\frac{2}{7}} \text { h }\left\{\mathrm{C}_{2},\left(P_{0 \mid 0}^{[1]}-P_{0 \mid 0}^{[2]}\right)\left(P_{1 \mid 0}^{[1] 2}\right)_{0} P_{1 \mid 0}^{[1]}\right\}_{2}-i 12 \sqrt{5} \text { h }\left\{\mathrm{C}_{2},\left(P_{0 \mid 0}^{[1]}-P_{0 \mid 0}^{[2]}\right)\left(P_{1 \mid 0}^{[1] 3}\right)_{3}\right\}_{2}+ \\
& \mathrm{h}^{2}\left(192 \sqrt{\frac{30}{7}}\left\{\hat{J}_{1}^{[0]}, \frac{\left(P_{1 \mid 0}^{[1] 2}\right)_{2}}{\left(P_{1 \mid 0}^{[1] 2}\right)_{0}}\right\}_{2}\left\langle\left(\left(P_{0 \mid 0}^{[1]} \cdot P_{0 \mid 0}^{[2]}\right)-\sqrt{3}\left(P_{1 \mid 0}^{[1] 2}\right)_{0}\right)\left(P_{1 \mid 0}^{[1] 2}\right)_{0}^{2}\right\rangle-\right. \\
& i 24 \sqrt{\frac{30}{7}}\left\{\hat{\mathcal{A}}_{1 \mid 0}, \frac{\left(P_{1 \mid 0}^{[1] 2}\right)_{2}}{\left(P_{1 \mid 0}^{[1] 2}\right)_{0}}\right\}_{2}\left\langle\left(7 \sqrt{3}\left(P_{0 \mid 0}^{[1]} \cdot P_{0 \mid 0}^{[2]}\right)+8\left(P_{1 \mid 0}^{[1] 2}\right)_{0}\right)\left(P_{1 \mid 0}^{[1] 2}\right)_{0}\right\rangle+ \\
& \left.i 192 \sqrt{\frac{30}{7}}\left\{\hat{\mathcal{B}}_{1 \mid 0}, \frac{\left(P_{1 \mid 0}^{[1] 2}\right)_{2}}{\left(P_{1 \mid 0}^{[1] 2}\right)_{0}}\right\}_{2}\left\langle\left(P_{0 \mid 0}^{[1]}-P_{0 \mid 0}^{[2]}\right)\left(P_{1 \mid 0}^{[1] 2}\right)_{0}^{2}\right\rangle\right),
\end{aligned}
$$

$\mathrm{dyn} 0 \mathrm{~b}=$

$\mathrm{h}^{2}$ times the content of the bracket () of the preceeding expression for dyn0a.

Now we make use of the previous specifications. We start with those for $\mathcal{K}_{1}$ and $\mathcal{K}_{2}$. By themselves, by Jacobi identities, and by the vanishing of the commutator $\left[P_{1 \mid 0}^{[1]}, P_{1 \mid 0}^{[1]}\right]_{1}$, the specifications imply 1.) the correctness of the previous hypothesis for the commutators $\left[\mathcal{K}_{q}, P_{1 \mid 0}^{[1]}\right]_{L}$ and 2.) the explicit form of the functions $\tau_{01}, \tau_{11}$ and $\tau_{23}$ :

$$
\tau_{01}=-\frac{8}{3} \sqrt{5}\left(P_{0 \mid 0}^{[1]} \cdot P_{0 \mid 0}^{[2]}\right), \quad \tau_{11}=-4 \sqrt{\frac{5}{3}}\left(P_{0 \mid 0}^{[1]} \cdot P_{0 \mid 0}^{[2]}\right), \quad \tau_{23}=0 .
$$

Next we turn to the specification for $\mathcal{C}_{2}$ in terms of $\mathcal{C}_{0}$ and $\mathcal{C}_{1}$. Working with the still unverified part of the hypothesis and inserting the complete expressions for the commutators $\left[\mathcal{K}_{q}, P_{1 \mid 0}^{[1]}\right]_{L}$ plus the specification for $\mathcal{C}_{2}$ into a scalar derivate of the generalized commutation relations from the second half of the previous list, we find the explicit expression for $\mathcal{C}_{0}$ :

$$
\mathcal{C}_{0}=-4 \sqrt{\frac{5}{3}} \text { h }\left\{\hat{\mathcal{B}}_{1 \mid 0}, P_{1 \mid 0}^{[1]}\right\}_{0}
$$

As a corrolary we obtain: $\left[\mathfrak{C}_{0}, P_{1 \mid 0}^{[1]}\right]_{1}=0$. Without further assistance of the derivates, this together with the previous specification for $\mathcal{C}_{2}$, the Jacobi identities and the vanishing of the commutator $\left[P_{1 \mid 0}^{[1]}, P_{1 \mid 0}^{[1]}\right]_{1}$ implies 1.) the self-consistency of the previous hypothesis for the commutators $\left[\mathcal{C}_{p}, P_{1 \mid 0}^{[1]}\right]_{L}$ and 2.) the explicit form of the "function" $\bar{\sigma}$ :

$$
\bar{\sigma}=3 \sqrt{\frac{6}{5}} .
$$


If we insert the explicit forms of $\tau_{01}, \tau_{11}$ and $\tau_{23}$ and the specifications for $\mathcal{C}_{2}, \mathcal{K}_{2}$ and $\mathcal{K}_{1}$ into the above formula for dyn0a, the contribution of all terms preceeding those inside the bracket ( ) at the end vanishes. Thereby we have established the equality of dyn0a and dyn0b which is part of our consistency check.

The numerical value $3 \sqrt{\frac{6}{5}}$ of $\bar{\sigma}$ and additional assistance of the derivates of the generalized commutation relations is required in order to show that the remaining dyn.a's are equal to the respective dyn.b's. Required are expressions for $\left[\mathcal{C}_{1}, \hat{\mathcal{A}}_{1 \mid 0}\right]_{1},\left[\mathcal{C}_{1}, \hat{\mathcal{A}}_{1 \mid 0}\right]_{2}$, $\left[\hat{t}_{1},\left(P_{0 \mid 0}^{[1]}-P_{0 \mid 0}^{[2]}\right)\right]_{1}$ and $\left[\mathcal{K}_{0}, \mathcal{C}_{1}\right]_{1}$. To illustrate the structure of these expressions we quote the ones for $\left[\hat{t}_{1},\left(P_{0 \mid 0}^{[1]}-P_{0 \mid 0}^{[2]}\right)\right]_{1}$ and $\left[\mathcal{K}_{0}, \mathcal{C}_{1}\right]_{1}$ in the appendix, the ones for $\left[\mathcal{C}_{1}, \hat{\mathcal{A}}_{1 \mid 0}\right]_{1}$ and $\left[\mathcal{C}_{1}, \hat{\mathcal{A}}_{1 \mid 0}\right]_{2}$ being equally lengthy if not even more so. In all cases the question whether the quantal composition laws $X_{2}^{[0]}=\ldots$ and $z_{2}^{[0]}=\ldots$ are in agreement with the generalized commutation relations has been answered affirmatively.

\section{Conclusions}

In the same way as selection rules limit the possible transition processes in atomic and elementary particle physics, (non-additive) composition laws in string physics restrict the possible outcome of the merging and splitting of strings. Within the (3+1)-dimensional Nambu-Goto theory we considered the subsector of bosonic closed strings in which all interactions proceed via string vertices, i.e. via merging and splitting.

In the classical setting two such tensorial non-additive composition laws had already been established [3]. In the first place, they had furnished numerical relations among the observables related to the three separate branches of the string vertex. On top of that, their rôle as generators for further non-additive composition laws by means of Poisson bracket operations had led to an attempt to count the number of new composition laws obtainable from them by such induction.

Based on this classical construction, we have explored the viability of suitably adapted versions of these composition laws in the corresponding quantum theory. In order to settle this issue, we looked for rigorous tests of the composition laws as relations in an associative graded algebra, its non-commutativity being curbed by the generalized commutation relations for the quantum observables related to the three separate branches of the vertex.

Apart from the obvious hermiticity, covariance and exchange symmetry requirements, the crucial tests entered in the form of two special types of constraints: 1.) a "kinematical constraint" in the context of manifest $O(3)$-covariance and 2.) several "dynamical constraints" probing the consistency of the quantized composition laws with the generalized commutation relations. The quantized composition laws passed all these tests unforcedly. Thus they can be regarded as a well-established structural element of the quantum theory of closed Nambu-Goto strings.

Now we are in a position to evaluate the additional mixed commutators of $X_{2}^{[0]}$ and $z_{2}^{[0]}$ on the one hand and of the generators of the algebras $\hat{\mathfrak{h}}^{[k]-}, k=1,2$, on the other hand - as far as the latter algebras and our explicit knowledge of them permit. The as yet undetermined functions $\xi$ and $\vartheta$ drop out of the pertinent calculations.

Further, due to the infinite dimension of the algebra of observables and in striking 
contrast to the Huygens-Newtonian laws of impact, by commutator induction we are in a position to produce more and more new quantized non-additive composition laws (which now in general do involve the functions $\xi$ and $\vartheta$ ). As we have already established, in this way we obtain all new non-additive composition laws (of the restricted type) within the $P$-linear span of $\hat{\mathfrak{V}}^{[k] 2}$.

Certainly, the functions $\xi$ and $\vartheta$ occurring on the r.h.s. of the composition laws $X_{2}^{[0]}=\ldots$ and $z_{2}^{[0]}=\ldots$, respectively, could be narrowed down by requiring i) covariance of the composition laws under Lorentz transformations, and ii) observance of the formal crossing symmetry (see above).

From the second requirement one could derive two linear inhomogeneous functional equations relating the functions $\xi$ and $\vartheta$ with arguments $\left(\frac{P_{0 \mid 0}^{[1]}-P_{0 \mid 0}^{[2]}}{\mathfrak{m}^{[0]}}\right)^{2}, \frac{\left(P_{1 \mid 0}^{[1]}\right)_{0}^{2}}{\left(\mathfrak{m}^{[0]}\right)^{2}}$ to the same functions with arguments $\left(\frac{P_{0 \mid 2}^{[1]}+P_{0 \mid 2}^{[2]}}{\mathfrak{m}^{[2]}}\right)^{2}, \frac{\left(P_{1 \mid 2}^{[1]^{2}}\right)_{0}}{\left(\mathfrak{m}^{2]}\right)^{2}}$. The inhomogeneities would be calculable from the double commutators arising in the course of the rearrangement of the composition laws $X_{2}^{[0]}=\ldots, Z_{2}^{[0]}=\ldots$ (for the real process: branch [1] merging with branch [2] to form branch [0]) into the composition laws $x_{2}^{[2]}=\ldots, z_{2}^{[2]}=\ldots$ (for the non-real process: branch [1] merging with crossed branch [0] to form crossed branch [2]).

This option will be pursued once arguments will have been presented that the formal crossing symmetry must also be observed in quantum theory.

Responsibilities: K.P. for the analytic line of reasoning and for the formulation of the manuscript, M.T. for the symbolic computations.

\section{Appendix}

$$
\begin{aligned}
& \text { dyn1a }= \\
& \frac{1}{\mathrm{~h}}\left\langle i \frac{12}{7 \sqrt{5}}\left\{\left[\mathcal{K}_{2}, \mathcal{C}_{2}\right]_{3}, \frac{\left(P_{1 \mid 0}^{[1] 3}\right)_{3}}{\left(P_{1 \mid 0}^{[1] 2}\right)_{0}}\right\}_{0}-i \frac{32}{35} \sqrt{\frac{2}{7}}\left\{\left[\mathcal{K}_{2}, \mathcal{C}_{2}\right]_{1}, P_{1 \mid 0}^{[1]}\right\}_{0}-i \frac{6}{7} \sqrt{\frac{2}{5}}\left\{\left[\mathcal{K}_{2}, \mathcal{C}_{1}\right]_{3}, \frac{\left(P_{1 \mid 0}^{[1] 3}\right)_{3}}{\left(P_{1 \mid 0}^{[1] 2}\right)_{0}}\right\}_{0}+\right. \\
& i \frac{4}{35} \sqrt{\frac{2}{21}}\left\{\left[\mathcal{K}_{2}, \mathcal{C}_{1}\right]_{1}, P_{1 \mid 0}^{[1]}\right\}_{0}-i \frac{22}{21} \sqrt{\frac{2}{5}}\left\{\left[\mathcal{K}_{1}, \mathcal{C}_{2}\right]_{3}, \frac{\left(P_{1 \mid 0}^{[1] 3}\right)_{3}}{\left(P_{1 \mid 0}^{[1] 2}\right)_{0}}\right\}_{0}-i \frac{4}{35} \sqrt{\frac{2}{21}}\left\{\left[\mathcal{K}_{1}, \mathcal{C}_{2}\right]_{1}, P_{1 \mid 0}^{[1]}\right\}_{0}- \\
& \left.i \frac{8}{7} \sqrt{\frac{2}{35}}\left\{\left[\mathcal{K}_{1}, \mathcal{C}_{1}\right]_{1}, P_{1 \mid 0}^{[1]}\right\}_{0}+i 4 \sqrt{\frac{2}{105}}\left\{\left[\mathcal{K}_{1}, \mathcal{C}_{0}\right]_{1}, P_{1 \mid 0}^{[1]}\right\}_{0}-i 4 \sqrt{\frac{2}{105}}\left\{\left[\mathcal{K}_{0}, \mathcal{C}_{1}\right]_{1}, P_{1 \mid 0}^{[1]}\right\}_{0}\right\rangle+ \\
& i \frac{128}{7} \sqrt{\frac{2}{105}}\left\{\left[\mathcal{K}_{2}, \hat{\mathcal{B}}_{1 \mid 0}\right]_{2},\left(P_{1 \mid 0}^{[1] 2}\right)_{2}\right\}_{0}-i \frac{128}{35} \sqrt{\frac{2}{7}}\left\{\left[\mathcal{K}_{1}, \hat{\mathcal{B}}_{1 \mid 0}\right]_{0},\left(P_{1 \mid 0}^{[1] 2}\right)_{0}\right\}_{0}- \\
& \frac{358}{21 \sqrt{35}}\left\{\left[\mathcal{C}_{2}, \hat{\mathcal{A}}_{1 \mid 0}\right]_{1}, P_{1 \mid 0}^{[1]}\right\}_{0}-\frac{922}{35 \sqrt{21}}\left\{\left[\mathcal{C}_{1}, \hat{\mathcal{A}}_{1 \mid 0}\right]_{1}, P_{1 \mid 0}^{[1]}\right\}_{0}+ \\
& \frac{4}{945 \sqrt{7}} \mathrm{~h}\left\{\mathcal{K}_{2},\left\langle 261 \sqrt{5}\left(P_{0 \mid 0}^{[1]}-P_{0 \mid 0}^{[2]}\right)-370 \sqrt{6}\left(P_{0 \mid 0}^{[1]}-P_{0 \mid 0}^{[2]}\right) \bar{\sigma}\right\rangle\left(P_{1 \mid 0}^{[1] 2}\right)_{2}\right\}_{0}- \\
& \frac{8}{945 \sqrt{7}} \mathrm{~h}\left\{\mathcal{K}_{0},\left\langle 1125\left(P_{0 \mid 0}^{[1]}-P_{0 \mid 0}^{[2]}\right)-2 \sqrt{30}\left(P_{0 \mid 0}^{[1]}-P_{0 \mid 0}^{[2]}\right) \bar{\sigma}\right\rangle\left(P_{1 \mid 0}^{[1] 2}\right)_{0}\right\}_{0}- \\
& \frac{32}{7} \mathrm{~h} \sqrt{\frac{2}{105}}\left\{\left[\hat{t}_{1},\left(P_{0 \mid 0}^{[1]}-P_{0 \mid 0}^{[2]}\right) \bar{\sigma}\right]_{1},\left(P_{1 \mid 0}^{[1] 2}\right)_{0} P_{1 \mid 0}^{[1]}\right\}_{0}+i \frac{2}{105} \sqrt{\frac{2}{7}} \mathrm{~h}\left\{\mathrm{C}_{1},\left\langle 44 \sqrt{15} \tau_{01}+20 \sqrt{5} \tau_{11}+\right.\right. \\
& \left.\left.4 \sqrt{15} \tau_{23}\left(P_{1 \mid 0}^{[1] 2}\right)_{0}+273 \sqrt{3}\left(P_{0 \mid 0}^{[1]} \cdot P_{0 \mid 0}^{[2]}\right)+216\left(P_{1 \mid 0}^{[1] 2}\right)_{0}\right\rangle P_{1 \mid 0}^{[1]}\right\}_{0}, \\
& \mathrm{dyn} 1 \mathrm{~b}=0
\end{aligned}
$$




$$
\begin{aligned}
& \operatorname{dyn} 2 \mathrm{~b}= \\
& \mathrm{h}^{2}\left(-48 \sqrt{\frac{14}{5}}\left\{\hat{J}_{1}^{[0]}, \frac{\left(P_{1 \mid 0}^{[1] 2}\right)_{2}}{\left(P_{1 \mid 0}^{[1] 2}\right)_{0}}\right\}_{2}\left\langle\left(P_{0 \mid 0}^{[1]}-P_{0 \mid 0}^{[2]}\right)\left(P_{1 \mid 0}^{[1] 2}\right)_{0}{ }^{3}\right\rangle-\right. \\
& i 144 \sqrt{\frac{42}{5}}\left\{\hat{\mathcal{A}}_{1 \mid 0}, \frac{\left(P_{1 \mid 0}^{[1] 2}\right)_{2}}{\left(P_{1 \mid 0}^{[1] 2}\right)_{0}}\right\}_{2}\left\langle\left(P_{0 \mid 0}^{[1]}-P_{0 \mid 0}^{[2]}\right)\left(P_{1 \mid 0}^{[1] 2}\right)_{0}{ }^{2}\right\rangle- \\
&\left.i 48 \sqrt{\frac{42}{5}}\left\{\hat{\mathcal{B}}_{1 \mid 0}, \frac{\left(P_{1 \mid 0}^{[1] 2}\right)_{2}}{\left(P_{1 \mid 0}^{[1] 2}\right)_{0}}\right\}_{2}\left\langle\left(\left(P_{0 \mid 0}^{[1]} \cdot P_{0 \mid 0}^{[2]}\right)+\sqrt{3}\left(P_{1 \mid 0}^{[1] 2}\right)_{0}\right)\left(P_{1 \mid 0}^{[1] 2}\right)_{0}{ }^{2}\right\rangle\right),
\end{aligned}
$$

$\mathrm{dyn} 3 \mathrm{~b}=$

$$
\begin{aligned}
& \mathrm{h}^{2}(-28704 \sqrt{3}\left\{\hat{J}_{1}^{[0]}, \frac{\left(P_{1 \mid 0}^{[1] 4}\right)_{4}}{\left(P_{1 \mid 0}^{[1] 2}\right)_{0}{ }^{2}}\right\}_{4}\left\langle\left(P_{0 \mid 0}^{[1]}-P_{0 \mid 0}^{[2]}\right)\left(P_{1 \mid 0}^{[1] 2}\right)_{0}{ }^{3}\right\rangle- \\
& i 107699\left\{\hat{\mathcal{A}}_{1 \mid 0}, \frac{\left(P_{1 \mid 0}^{[1] 4}\right)_{4}}{\left(P_{1 \mid 0}^{[1] 2}\right)_{0}{ }^{2}}\right\}_{4}\left\langle\left(P_{0 \mid 0}^{[1]}-P_{0 \mid 0}^{[2]}\right)\left(P_{1 \mid 0}^{[1] 2}\right)_{0}{ }^{2}\right\rangle+ \\
&\left.i 96\left\{\hat{\mathcal{B}}_{1 \mid 0}, \frac{\left(P_{1 \mid 0}^{[1] 4}\right)_{4}}{\left(P_{1 \mid 0}^{[1] 2}\right)_{0}^{2}}\right\}_{4}\left\langle\left(19\left(P_{0 \mid 0}^{[1]} \cdot P_{0 \mid 0}^{[2]}\right)-373 \sqrt{3}\left(P_{1 \mid 0}^{[1] 2}\right)_{0}\right)\left(P_{1 \mid 0}^{[1] 2}\right)_{0}^{2}\right\rangle\right), \\
& {\left[\hat{t}_{1},\left(P_{0 \mid 0}^{[1]}-P_{0 \mid 0}^{[2]}\right)\right]_{1}=-i \frac{64}{63} \sqrt{2} \mathcal{C}_{1}-i \frac{5}{63} \sqrt{10}\left\{\mathcal{C}_{1}, \frac{\left(P_{1 \mid 0}^{[1] 2}\right)_{2}}{\left(P_{1 \mid 0}^{[1] 2}\right)_{0}}\right\}_{1}-i \frac{4}{7} \sqrt{30}\left\{\hat{\mathcal{B}}_{1 \mid 0}, P_{1 \mid 0}^{[1]}\right\}_{1}, } \\
& {\left[\mathcal{K}_{0}, \mathcal{C}_{1}\right]_{1}=i \sqrt{\frac{6}{5}}\left\{\mathcal{K}_{0},\left(P_{0 \mid 0}^{[1]}-P_{0 \mid 0}^{[2]}\right) P_{1 \mid 0}^{[1]}\right\}_{1}+\frac{44}{21} \sqrt{\frac{5}{3}}\left\{\mathcal{C}_{1},\left(P_{1 \mid 0}^{[1] 2}\right)_{0}\right\}_{1}+\frac{100}{21 \sqrt{3}}\left\{\mathcal{C}_{1},\left(P_{1 \mid 0}^{[1] 2}\right)_{2}\right\}_{1}-} \\
& \frac{8}{3} \sqrt{5}\left\{\mathcal{C}_{1},\left(P_{0 \mid 0}^{[1]} \cdot P_{0 \mid 0}^{[2]}\right)\right\}_{1}-i \frac{40}{3}\left\{\hat{J}_{1}^{[0]}, P_{1 \mid 0}^{[1]}\right\}_{1}\left\langle\left(P_{0 \mid 0}^{[1]}-P_{0 \mid 0}^{[2]}\right)\left(P_{1 \mid 0}^{[1] 2}\right)_{0}\right\rangle+ \\
& \frac{56}{3 \sqrt{3}}\left\{\hat{\mathcal{A}}_{1 \mid 0}, P_{1 \mid 0}^{[1]}\right\}_{1}\left\langle\left(P_{0 \mid 0}^{[1]}-P_{0 \mid 0}^{[2]}\right)\right\rangle-\frac{16}{63} \sqrt{3}\left\{\hat{\mathcal{B}}_{1 \mid 0}, P_{1 \mid 0}^{[1]}\right\}_{1}\left\langle 35\left(P_{0 \mid 0}^{[1]} \cdot P_{0 \mid 0}^{[2]}\right)+4 \sqrt{3}\left(P_{1 \mid 0}^{[1] 2}\right)_{0}\right\rangle .
\end{aligned}
$$

\section{References}

[1] K. Pohlmeyer, Ann. Phys. (Leipzig) 8, 19 (1999),

[2] K. Pohlmeyer, Mod. Phys. Lett. A 10, 295 (1995),

[3] J. Großer, "Kompositionsgesetze für die invarianten Ladungen des Nambu-GotoStrings", Diplomarbeit, Fakultät für Physik, Universität Freiburg (1999),

[4] C.U. Condon and G.H. Shortley, The Theory of Atomic Spectry, Cambridge University Press (1935),

[5] K. Pohlmeyer and K.-H. Rehren, Commun. Math. Phys. 105, 593 (1986); in particular: p. 615.

[6] M. Rotenberg, R. Bivins, N. Metropolis, and J. K. Wooten (Jr.), The 3-j and 6-j Symbols, The Technology Press, Massachusetts Institute of Technology, Cambridge (Massachusetts) 1959, p. 15 